\title{
Ultrawideband Noise Radar Tomography: Principles, Simulation, and Experimental Validation
}

\author{
Hee Jung Shin, ${ }^{1}$ Ram M. Narayanan, ${ }^{1}$ Mark A. Asmuth, ${ }^{1}$ and Muralidhar Rangaswamy ${ }^{2}$ \\ ${ }^{1}$ Pennsylvania State University, University Park, PA 16802, USA \\ ${ }^{2}$ Air Force Research Laboratory, WPAFB, Dayton, OH 45433, USA \\ Correspondence should be addressed to Ram M. Narayanan; ram@engr.psu.edu
}

Received 2 November 2015; Accepted 15 March 2016

Academic Editor: Walter De Raedt

Copyright (c) 2016 Hee Jung Shin et al. This is an open access article distributed under the Creative Commons Attribution License, which permits unrestricted use, distribution, and reproduction in any medium, provided the original work is properly cited.

\begin{abstract}
The paper introduces the principles, simulation results, and hardware implementation of ultrawideband (UWB) noise radar for obtaining tomographic images of various scenarios of rotating cylindrical objects using independent and identically distributed UWB noise waveforms. A UWB noise radar was designed to transmit multiple UWB random noise waveforms over the 3-5 GHz frequency range and to measure the backward scattering data for the validation of the theoretical analysis and numerical simulation results. The reconstructed tomographic images of the rotating cylindrical objects based on experimental results are seen to be in good agreement with the simulation results, which demonstrates the capability of UWB noise radar for complete two-dimensional tomographic image reconstruction of various shaped metallic and dielectric target objects.
\end{abstract}

\section{Introduction}

Noise radar has been developed and refined for covert detection and imaging applications over the past few decades due to several advantages, such as low probability of interception (LPI) [1-3], electronic counter-countermeasure (ECCM) [46], and relatively simple hardware configurations [7-9]. Further, ultrawideband (UWB) noise radar has achieved high-resolution detection and imaging in foliage penetration (FOPEN) imaging [10, 11], through-wall imaging (TWI) [12$14]$, and ground penetration radar (GPR) imaging $[15,16]$ by employing an ultrawide bandwidth. For obtaining high-resolution images of various shaped targets, tomographic radar imaging is emerging as a powerful imaging technique [17-19]. In general, radar imaging techniques tend to be formulated in the time domain to use computationally efficient backprojection algorithms and to provide accurate object shape and location results of the target objects [20-22]. On the other hand, the objective of tomography-based radar imaging is to characterize the material property profiles of the target objects from the scattered fields and reconstruct specific scatterers within the interrogation medium by solving the inverse scattering problem [23-26].
Practical tomography systems have been developed and implemented in a way that the multidimensional images of the target object are successfully obtained from electromagnetic scattering with various waveforms [27-29]. The requirement when using this method is the need for mechanical rotation of either the body or the antenna in order to obtain measurements in different views [30-32]. Every object, when inserted into an electromagnetic field, causes a well-defined field change, and diffraction occurs when the wavelength of the radiation is of the order of the dimension of the object. A one-to-one relationship relating the scattered field to the object complex permittivity can be obtained within the Born approximation, via a Fourier transform using the so-called Fourier diffraction theorem [33-35].

This paper introduces the principle, simulation, and experiment results of tomographic imaging of various target scenarios using diffraction tomography for a bistatic UWB noise radar. Since the frequency behavior of the electrical parameters of the target objects due to the illuminating electromagnetic fields depends on the frequency used in the system, UWB imaging clearly provides advantages over single or narrow band frequency operation in terms of resolution and 


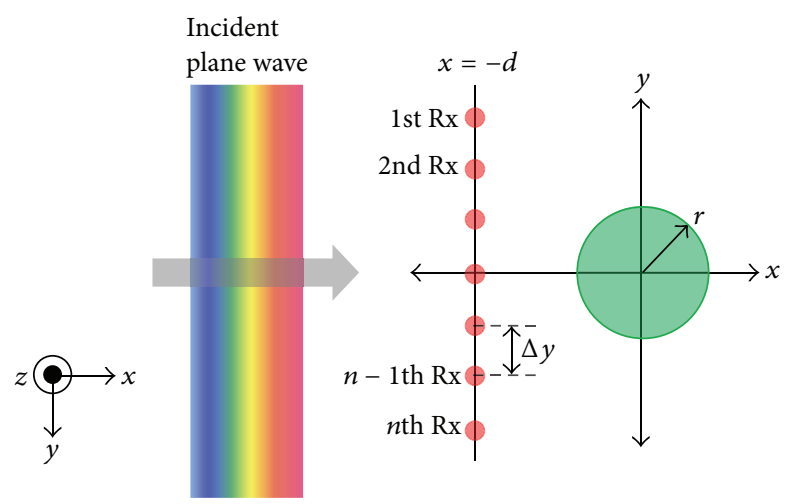

FIGURE 1: Two-dimensional backward scattering geometry for a cylindrical object. The $z$-polarized incident wave is illuminated from $-x$ direction and propagated in free space.

accuracy. Furthermore, transmitting random noise waveform inherits the aforementioned advantages of noise radar. The paper is organized as follows. In Section 2, theoretical analysis of the image reconstruction for a bistatic noise radar system with two-dimensional scattering geometry using Fourier diffraction theorem under the assumption of plane wave illumination is presented. The numerical simulation results of diffraction tomography using UWB random noise waveforms are presented in Section 3. Section 4 describes the RF hardware implementation and experimental results from that noise radar system. Further, we compare the images obtained with simulations and experiments. Conclusions are drawn in Section 5 .

\section{Theory and Formulation}

The main advantage of transmitting a random noise waveform is to covertly detect and image a target without alerting the presence of radar system. Such LPI characteristic of the noise radar is achieved by using a random noise waveform, which is constantly varying and never repeats itself exactly $[36,37]$. The random noise waveform herein is mathematically defined as discrete time wide-sense stationary (WSS) and ergodic random process and a sequence of independent and identically distributed (i.i.d.) random variable drawn from a normal distribution, $\mathcal{N}\left(0, \sigma^{2}\right)$. The power spectral density (PSD) of white Gaussian noise (WGN) waveform is ideally a nonzero constant for all frequencies. In practical situations, however, the PSD of the random noise waveform may not be uniform across the wide frequency ranges because a finite number of random noise amplitude samples must be chosen for WGN waveform generation. In order to bypass such a shortcoming, multiple sequences of i.i.d. noise waveforms are transmitted to flatten the spectral density without changing the mean and variance [38].

Figure 1 shows two-dimensional backward scattering geometry for a cylindrical target object. The values of receiver spacing, $\Delta y$, and the frequency sampling interval, $\Delta f$, are determined based on the maximum operating frequency and the size of the object, respectively, to ensure an image that is free of false aliases [39-41].
The analysis starts from a single frequency and develops the process for UWB by summing up the analysis results for $N$ discrete frequencies. As shown in Figure 1, the incident $z$ polarized plane wave of the WGN waveform for $N$ multiple frequencies takes the form

$$
\begin{aligned}
& E_{i}\left(k_{n}, \vec{r}\right) \\
& \quad=\widehat{z}\left(E_{1} e^{-j k_{1} \hat{x} \cdot \vec{r}}+E_{1} e^{-j k_{2} \hat{x} \cdot \vec{r}}+\cdots+E_{N} e^{-j k_{N} \widehat{x} \cdot \vec{r}}\right) \\
& \quad=\widehat{z} \sum_{n=1}^{N} E_{n} e^{-j k_{n} \hat{x} \cdot \vec{r}}, \\
& H_{i}\left(k_{n}, \vec{r}\right)=-\widehat{y} \frac{1}{\eta} \times E_{i}\left(k_{n}, \vec{r}\right),
\end{aligned}
$$

where $k_{n}=\omega_{n} / c$ is the wavenumber, $\eta$ is the intrinsic impedance in free space, and $E_{n}$ is the field amplitude of the transmitted white Gaussian noise waveform at each discrete frequency of interest. We start the analysis from a single frequency and develop the process for $N$ multiple frequencies by summing up the analysis results for $N$. The entire analysis must be repeated for $K$ times when $K$ multiple sequences of i.i.d. noise waveforms are transmitted.

Consider a dielectric target object illuminated by an incident plane wave denoted in (1), which induces an equivalent electric current distribution, given by

$$
J_{\text {eq,dielectric }}\left(k_{n}, \vec{r}\right)=j \omega\left[\varepsilon(\vec{r})-\varepsilon_{0}\right] E\left(k_{n}, \vec{r}\right),
$$

where $\varepsilon(\vec{r})$ and $\varepsilon_{0}$ denote the complex permittivity of the object and the vacuum permittivity, respectively. $E\left(k_{n}, \vec{r}\right)$ in (2) is the total field due to the presence of the object and is expressed as the sum of the incident field $E_{i}\left(k_{n}, \vec{r}\right)$ and the scattered field $E_{s}\left(k_{n}, \vec{r}\right)$; that is,

$$
E\left(k_{n}, \vec{r}\right)=E_{i}\left(k_{n}, \vec{r}\right)+E_{s}\left(k_{n}, \vec{r}\right) .
$$

If the object is assumed to be a perfect electric conductor (PEC), the equivalent induced current distribution is calculated by applying the physical optics approximation [42] and is given by

$$
J_{\mathrm{eq}, \mathrm{PEC}}\left(k_{n}, \vec{r}\right)=2 \widehat{n}(\vec{r}) \times H_{i}\left(k_{n}, \vec{r}\right),
$$


TABLE 1: Target objects imaged by the numerical simulations.

\begin{tabular}{lccc}
\hline Object material & Object description & Dimension \\
\hline \multirow{2}{*}{ PEC } & Circular cylinder & $20 \mathrm{~cm}$ diameter $\left(7.9^{\prime \prime}\right.$ diameter $)$ & \\
& Square box & $20 \mathrm{~cm} \times 20 \mathrm{~cm}\left(7.9^{\prime \prime} \times 7.9^{\prime \prime}\right)$ & \\
& Triangular prism & $20 \mathrm{~cm}$ side $\left(7.9^{\prime \prime}\right.$ side $)$ & Height of the objects is assumed to be infinitely long \\
Dielectric $\varepsilon_{r}=5.8-j 0.75$ & Circular cylinder & $16 \mathrm{~cm}$ diameter $\left(6.3^{\prime \prime}\right.$ diameter $)$ & \\
& Square box & $16 \mathrm{~cm} \times 16 \mathrm{~cm}\left(6.3^{\prime \prime} \times 6.3^{\prime \prime}\right)$ & \\
\hline
\end{tabular}

where $\widehat{n}(\vec{r})$ is the outward unit normal vector to the boundary of scatterer.

The general form of the scattered field $E_{s}\left(k_{n}, \vec{r}\right)$ created by the equivalent induced current is written as

$$
E_{s}\left(k_{n}, \vec{r}\right)=-j \omega \mu_{0} \int_{S} J_{\mathrm{eq}}\left(k_{n}, \vec{r}^{\prime}\right) \cdot G\left(\vec{r}-\vec{r}^{\prime}\right) d \vec{r}^{\prime}
$$

where $S$ is the boundary of scatterer and $G\left(\vec{r}-\vec{r}^{\prime}\right)$ is the twodimensional Green's function.

Based on [43], the $z$-polarized scattered field at the receivers $x=-d$ can be written as

$$
\begin{gathered}
u_{s}\left(k_{n}, x=-d, y\right)=\widehat{z} \cdot E_{s}\left(k_{n}, x=-d, y\right) \\
=\psi \iint o\left(\vec{r}^{\prime}\right) e^{-j k_{n} \hat{x} \cdot \vec{r}^{\prime}} G\left(\vec{r}-\vec{r}^{\prime}\right) d^{2} \vec{r}^{\prime},
\end{gathered}
$$

where $\psi=k_{n}^{2} E_{n}$ for the dielectric object and $\psi=j k_{n} E_{n}$ for the PEC object case. $o\left(\vec{r}^{\prime}\right)$ is the scattering object function which is related to the shape of the target object. By using the plane wave expansion of Green's function, the onedimensional Fourier transform of the scattered field is given by $[44,45]$

$$
\widetilde{U}_{s}\left(k_{n}, x=-d, k_{y}\right)=\frac{-j \psi}{2 \gamma} e^{-j \gamma d} \widetilde{O}\left(-\gamma-k_{n}, k_{y}\right),
$$

where

$$
\gamma= \begin{cases}\sqrt{k_{n}{ }^{2}-k_{y}{ }^{2}} & \text { as }\left|k_{y}\right| \leq k_{n} \\ -j \sqrt{k_{y}{ }^{2}-k_{n}^{2}} & \text { as }\left|k_{y}\right|>k_{n} .\end{cases}
$$

In deriving (7), the one-dimensional Fourier transform of $\widetilde{U}_{s}$ in the $y$-direction is defined as

$$
\widetilde{U}_{s}\left(k_{n}, x=-d, k_{y}\right)=\int u_{s}\left(k_{n}, x=-d, y\right) e^{j k_{y} y} d y .
$$

If we define $k_{x}=-\gamma-k_{n}$, the scattering object function in the Fourier domain $\widetilde{O}\left(k_{x}, k_{y}\right)$ is the two-dimensional Fourier transform of the scattering object function such that

$$
\widetilde{O}\left(k_{x}, k_{y}\right)=\iint o(x, y) e^{-j\left(k_{x} x+k_{y} y\right)} d x d y .
$$

The image of the target object is constructed by calculation of the magnitude of scattering object function $o(x, y)$ in rectangular coordinate after performing two-dimensional inverse Fourier transform of (10). By using $N$ number of frequencies, the resolution and accuracy of the image can be enhanced. Also the variance of the frequency response in WGN is reduced by taking average of the frequency responses of $K$ multiple transmissions of i.i.d. noise waveforms [46, 47].

As the object is sequentially rotated with respect to the rotational axis for the value of rotational step angle $\theta$, the corresponding scattering object function at each angular increment $\theta$ is obtained by rotating the obtained scattering object function $o(x, y)$ with respect to the corresponding rotational step angle $\theta$ in $x$ - $y$ image coordinates. A block diagram shown in Figure 2 displays the tomographic image reconstruction procedure for $K$ multiple transmitted i.i.d. noise waveforms for a sequentially rotated object using diffraction tomography.

\section{Numerical Simulation Results}

As shown in Figure 2, the complete tomographic image of the target object is reconstructed based on the number of transmitted noise waveforms and angular steps. The image quality of UWB noise radar tomography has been quantitatively analyzed by calculating the cumulative mean squared error (MSE) between the corresponding pixels of the reference images and the reconstructed images $[48,49]$.

For scattered field calculations and diffraction tomography simulations with multiple i.i.d. WGN waveforms, 10 bandlimited UWB noise waveforms over a frequency range from $3 \mathrm{GHz}$ to $5 \mathrm{GHz}$ are generated with 500 random amplitude samples drawn from $\mathcal{N}\left(0, \sigma^{2}\right)$ and are sequentially transmitted for backward scattered field calculations. Also, the rotational step angle $\theta$ is set to $9^{\circ}$ based on the meansquared-error (MSE) analysis [50]. For each transmitted noise waveform, 40 discrete images are obtained after full $360^{\circ}$ rotation for each transmitted noise waveform, and the complete tomographic image of the target is obtained by combining these 40 discrete images into one single image.

Table 1 is a list of target objects for the numerical simulations. The dielectric objects are assumed to be a homogeneous concrete block; the real and imaginary parts of complex relative permittivity are assumed to be 5.8 and 0.75 , respectively, at the evaluation frequency of $3 \mathrm{GHz}$ [51]. The moisture contents and surface roughness of the dielectric objects are not considered for the simulations.

Our simulation results below show cases wherein 1, 3, 7, and 10 noise waveforms are averaged. In an earlier paper [43], we compared the averaged image with that obtained using the commonly used first derivative Gaussian waveform and determined that the mean squared error (MSE) between the images dropped off exponentially as the number of averages 


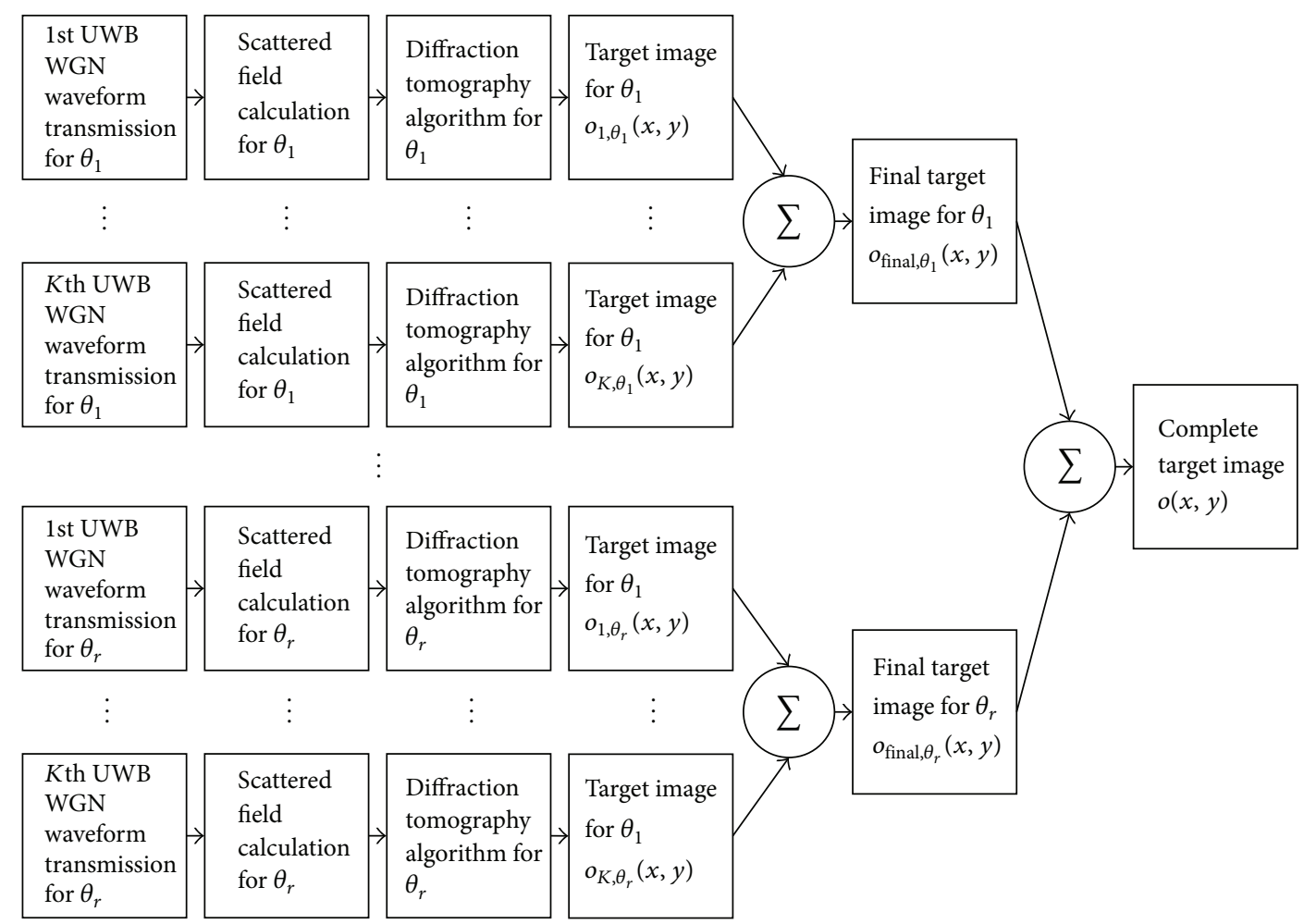

FIGURE 2: The image reconstruction procedure with $K$ multiple i.i.d. WGN transmitted waveforms for a sequentially rotated object using diffraction tomography.

increased. The MSE tended to level off after about 6 averages, after which the drop in MSE was at a slower rate. Therefore, we considered 10 averages to be adequate for generating the final image.

3.1. Circular PEC Cylinder. Figure 3 shows a bistatic noise radar system with two-dimensional scattering geometry for a circular PEC cylinder. The cylinder with a radius of $10 \mathrm{~cm}$ is located at $(0 \mathrm{~cm}, 0 \mathrm{~cm})$, and its height is assumed to be infinitely long along the $z$-axis. A linear array of 55 receivers is positioned $68 \mathrm{~cm}$ away from the center of the object with the receiver spacing $\Delta y$ of $1.5 \mathrm{~cm}$ in order to avoid any aliasing. The scattered field is uniformly sampled at receiving array $\mathrm{Rx}$ 1 through $\mathrm{Rx} 55$ with frequency swept within the frequency ranges of $3-5 \mathrm{GHz}$. When the simulation is completed, the object is rotated in clockwise direction in steps of $9^{\circ}$ with respect to the origin $(0 \mathrm{~cm}, 0 \mathrm{~cm})$, and the simulation process was repeated until the object was fully rotated over $360^{\circ}$.

Four tomographic images of the circular PEC cylinder with 10 transmitted i.i.d. bandlimited WGN waveforms are shown in Figures 4(a), 4(b), 4(c), and 4(d) when 1, 3, 7, and 10 noise waveforms are transmitted, respectively. Complete tomographic imaging of the cylinder is successfully achieved after averaging all ten images by visual inspection of the formed images as shown in Figure 4(d), and increasing the number of transmissions of the i.i.d. UWB WGN waveform enhances the quality of final tomographic image of the object by reducing the variance of the spectral response of WGN.
3.2. Square PEC Box. Figure 5 shows a bistatic noise radar system with two-dimensional scattering geometry for a square PEC box. The length of each side of the square $(a)$ is $20 \mathrm{~cm}$, and its height is assumed to be infinitely long along the $z$-axis. Other simulation parameters, such as the position of receivers, the parameters for receiver spacing, and the rotational angle, are identical to the circular PEC cylinder case. In order to generate the complete tomographic image of the square PEC box shown in Figure 5, 10 i.i.d. random noise waveforms are sequentially transmitted as well.

Figures 6(a), 6(b), 6(c), and 6(d) display four tomographic images of the square PEC box when $1,3,7$, and 10 noise waveforms are transmitted, respectively. As seen in Figure 6, four corners of the square box are not clearly imaged and fringes are observed at the sharp edges, as expected, affecting the image quality of the formed images. However, the approximate square shape can easily be inferred from the image.

3.3. Equilateral Triangular PEC Prism. A bistatic noise radar system with two-dimensional scattering geometry for an equilateral triangular PEC prism is shown in Figure 7. The prism is located at $(0 \mathrm{~cm}, 0 \mathrm{~cm})$, and the length of each side of the triangle $(a)$ is $20 \mathrm{~cm}$. Similarly, the target object is considered as an infinitely long prism along the $z$-axis. All simulation parameters remain unchanged.

Four tomographic images of the triangular PEC prism with 10 transmitted i.i.d. bandlimited WGN waveforms are shown in Figures 8(a), 8(b), 8(c), and 8(d) when 1, 3, 7, 


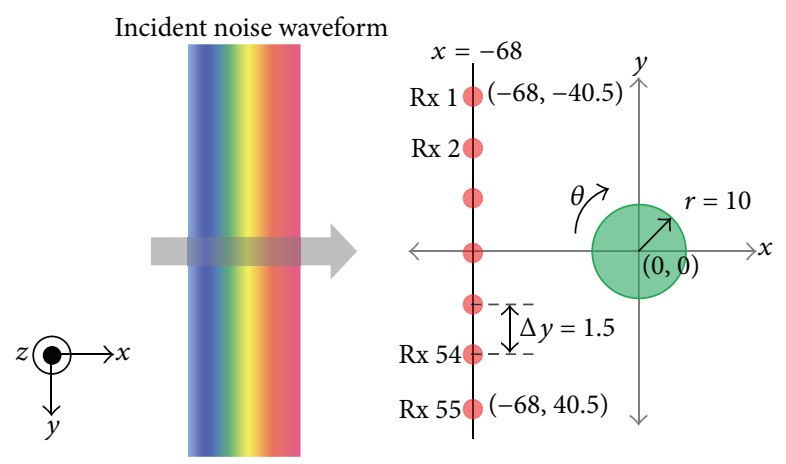

FIGURE 3: A bistatic noise radar system with two-dimensional backward scattering geometry for a circular PEC cylinder. The target object is surrounded by vacuum. The rotational step angle is $9^{\circ}$.

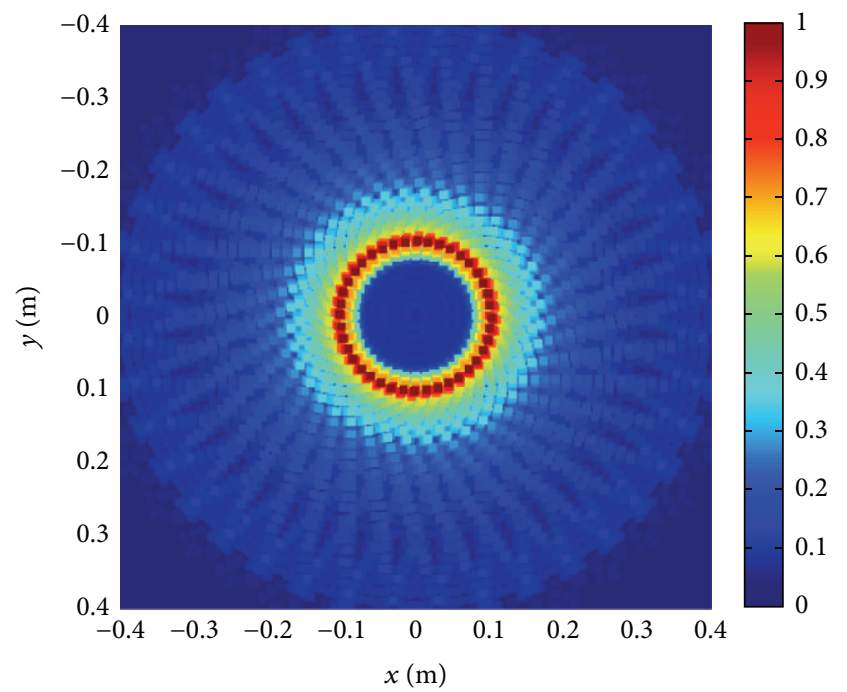

(a)

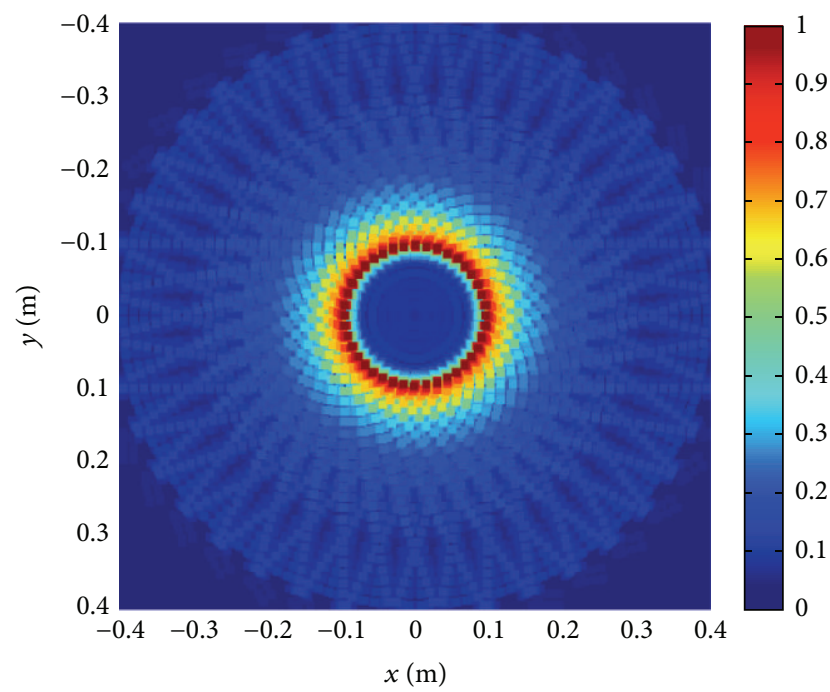

(c)

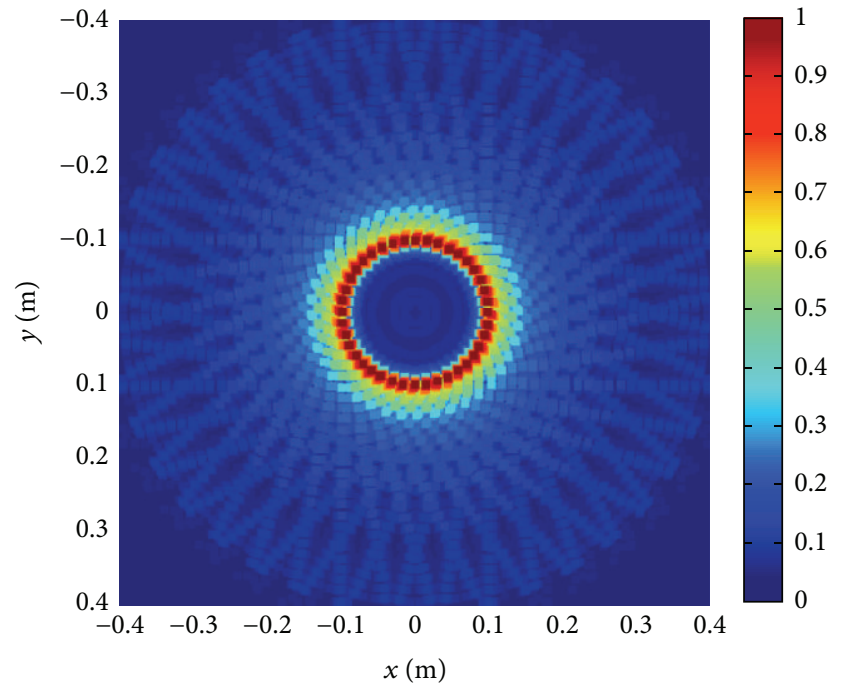

(b)

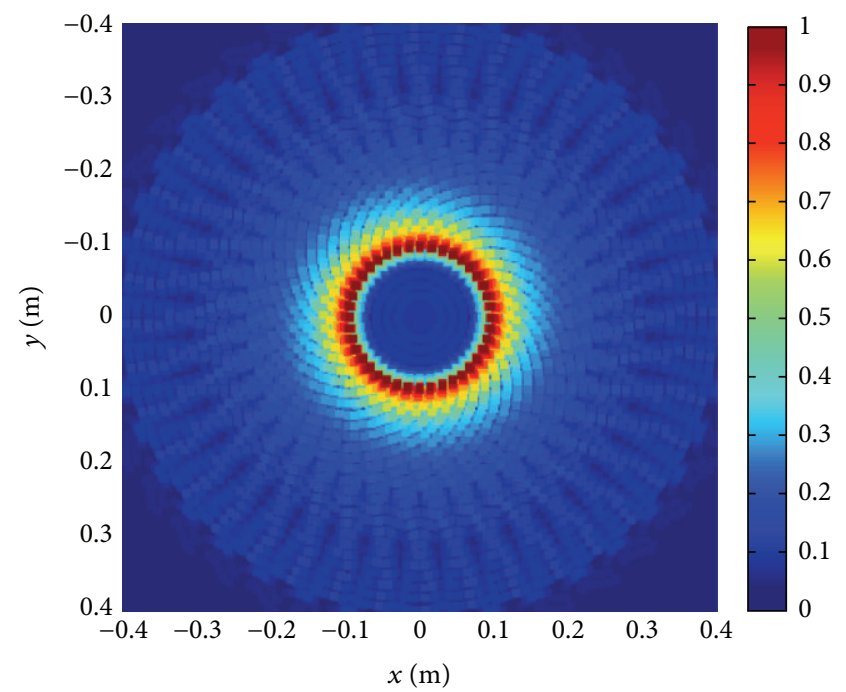

(d)

FIGURE 4: Complete tomographic image of a PEC cylinder with a radius of $10 \mathrm{~cm}$ located at $(0 \mathrm{~cm}, 0 \mathrm{~cm})$ after summing and averaging process based on (a) one transmitted WGN waveform, (b) three transmitted WGN waveforms, (c) seven transmitted WGN waveforms, and (d) all ten transmitted WGN waveforms. 


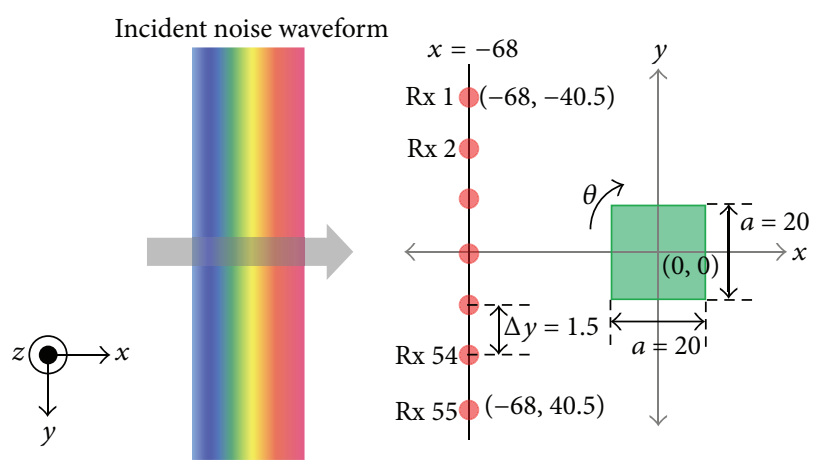

FIGURE 5: A bistatic noise radar system with two-dimensional backward scattering geometry for a square PEC box. The target object is surrounded by vacuum. The rotational step angle is $9^{\circ}$.

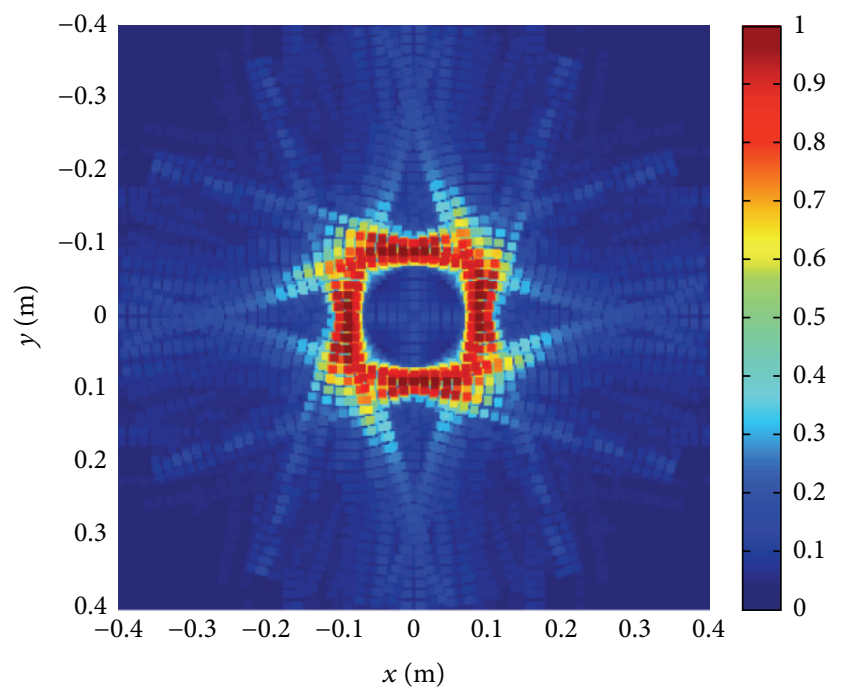

(a)

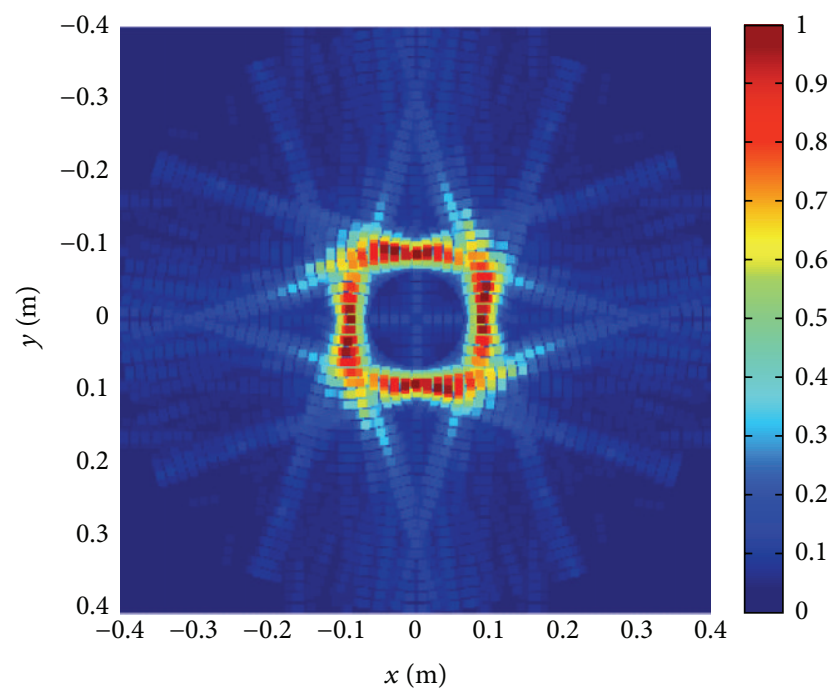

(c)

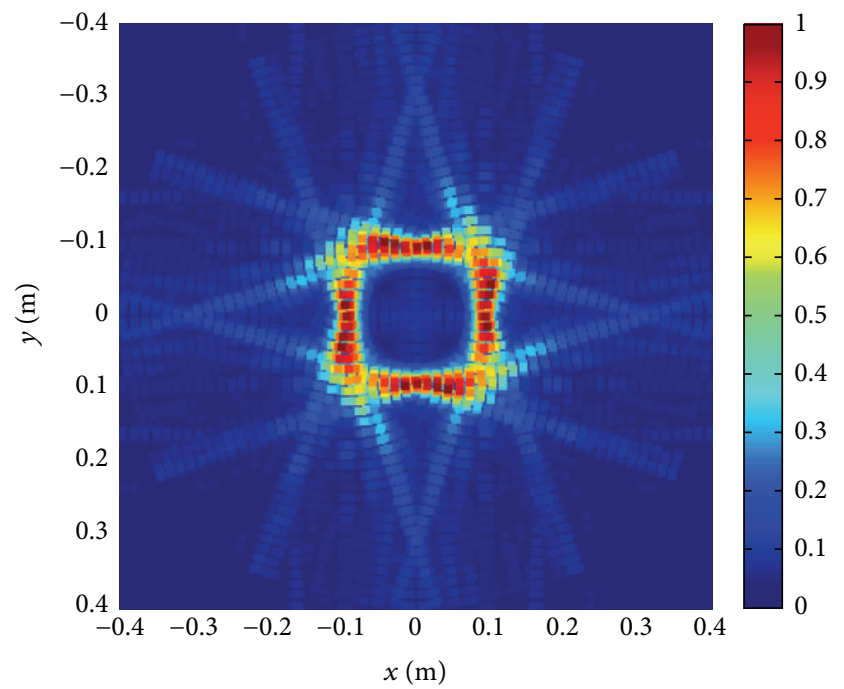

(b)

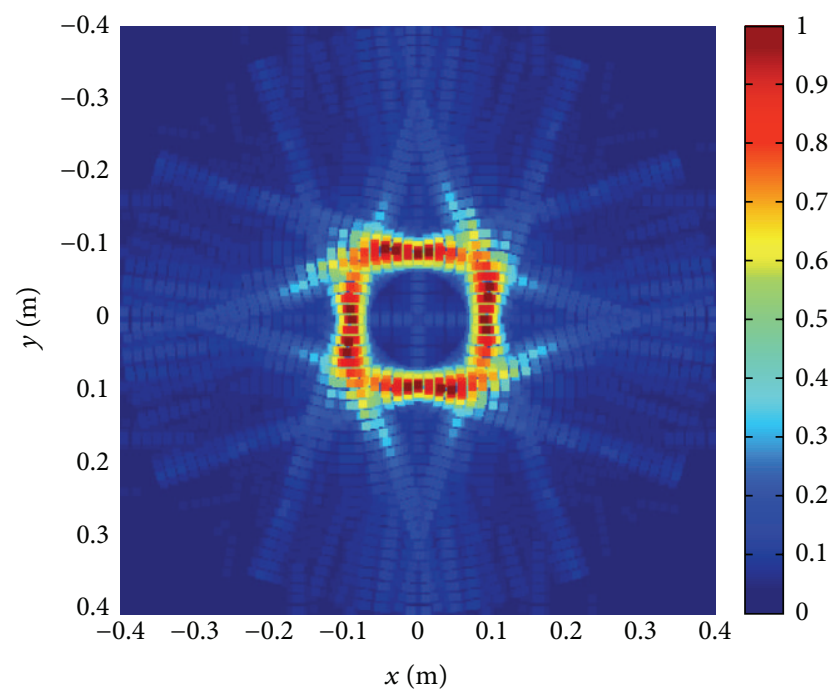

(d)

FIGURE 6: Complete tomographic image of a square PEC box with a length of $20 \mathrm{~cm}$ located at $(0 \mathrm{~cm}, 0 \mathrm{~cm})$ after summing and averaging process based on (a) one transmitted WGN waveform, (b) three transmitted WGN waveforms, (c) seven transmitted WGN waveforms, and (d) all ten transmitted WGN waveforms. 


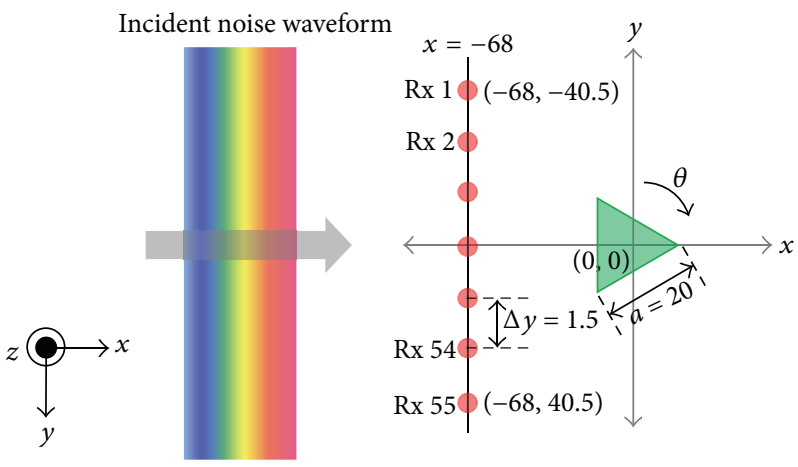

FIGURE 7: A bistatic noise radar system with two-dimensional backward scattering geometry for an equilateral triangular PEC prism. The target object is surrounded by vacuum. The rotational step angle is $9^{\circ}$.

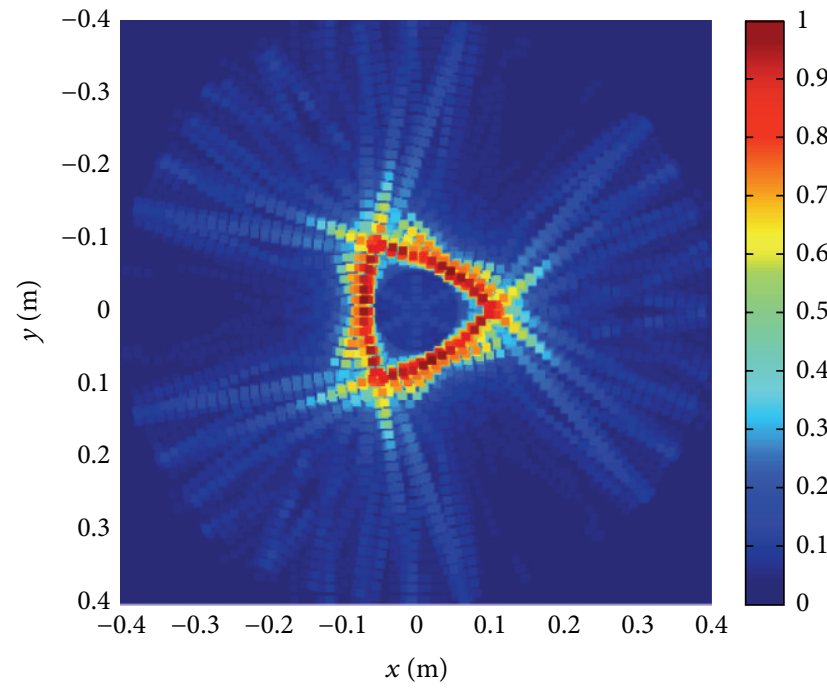

(a)

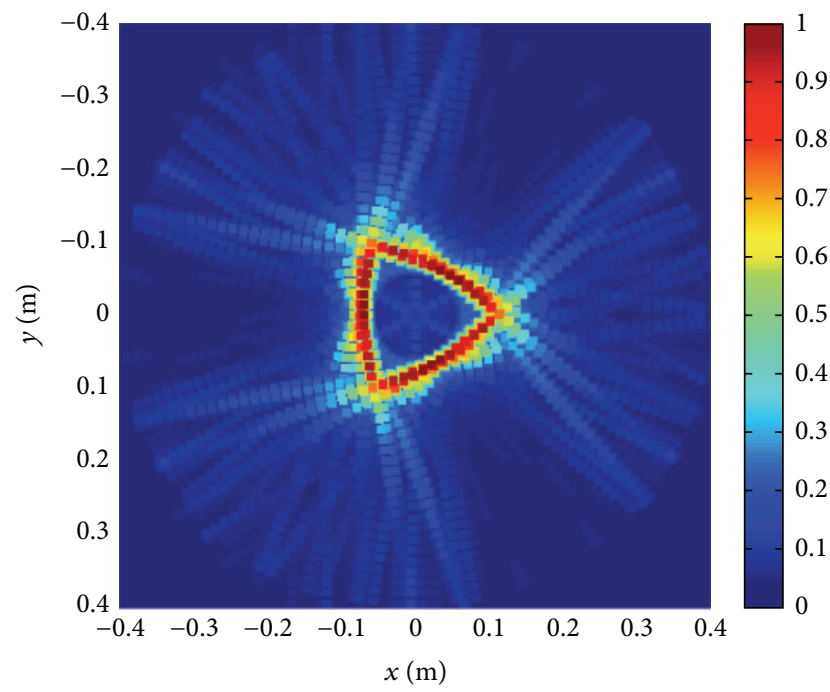

(c)

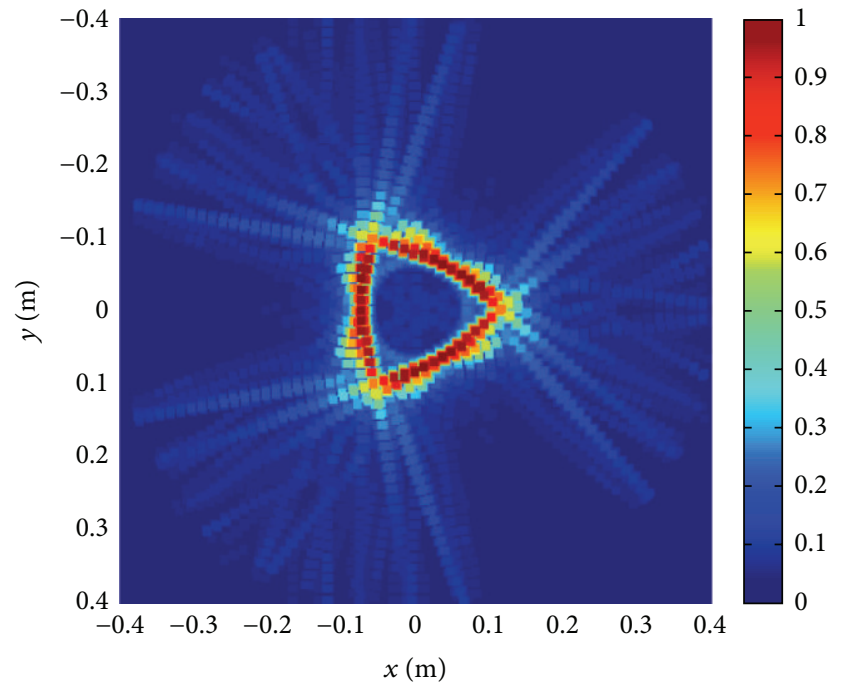

(b)

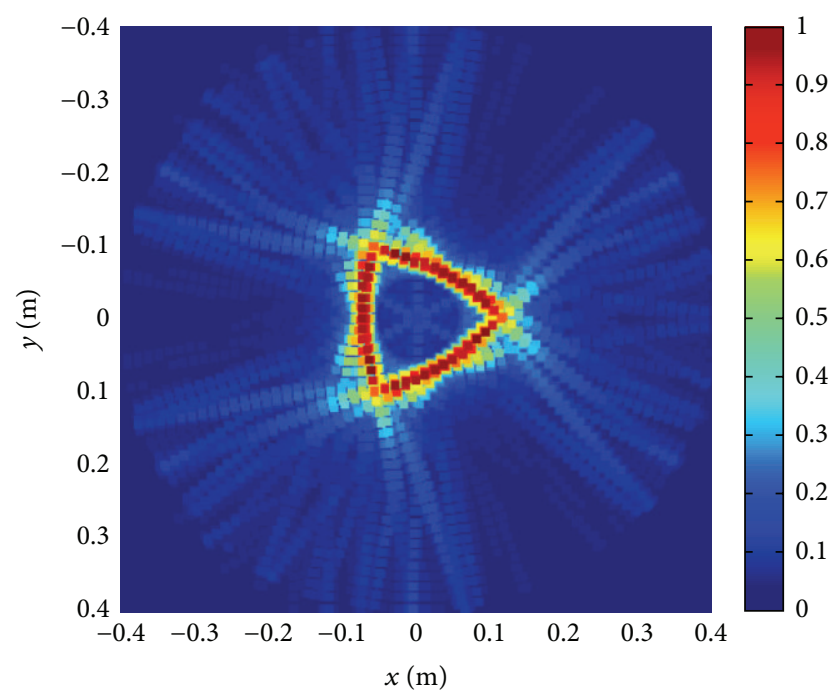

(d)

FIGURE 8: Complete tomographic image of an equilateral triangular PEC prism with a length of $20 \mathrm{~cm}$ located at $(0 \mathrm{~cm}, 0 \mathrm{~cm})$ after summing and averaging process based on (a) one transmitted WGN waveform, (b) three transmitted WGN waveforms, (c) seven transmitted WGN waveforms, and (d) all ten transmitted WGN waveforms. 


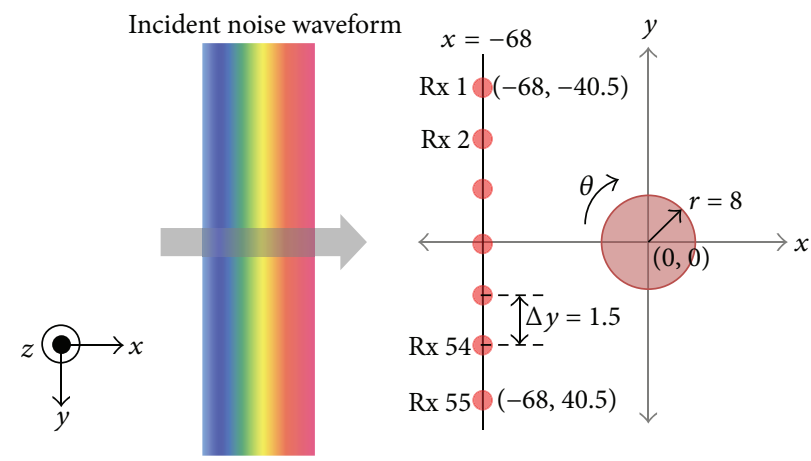

FIGURE 9: A bistatic noise radar system with two-dimensional backward scattering geometry for a circular PEC cylinder. The target object is surrounded by vacuum. The rotational step angle is $9^{\circ}$.

and 10 noise waveforms are transmitted, respectively. The image quality of complete tomographic image of the prism is enhanced as the number of transmitted noise waveforms increases. Although fringes are evident at the corners of the prism due to the scattering at the sharp edges, the approximate shape of the object is clearly observed.

3.4. Circular Dielectric Cylinder. Figure 9 shows a bistatic noise radar system with two-dimensional scattering geometry for a circular dielectric cylinder. As previously discussed, the dielectric cylinder is assumed to be a homogeneous concrete material, with the real and imaginary parts of complex relative permittivity set to 5.8 and 0.75 , respectively. The aforementioned physical conditions of the object, such as moisture contents and surface roughness, are not considered for the simulations.

As shown in Figure 9, the dielectric cylinder with a radius of $8 \mathrm{~cm}$ is located at $(0 \mathrm{~cm}, 0 \mathrm{~cm})$, and the height of the cylinder is assumed to be infinitely long along the $z$-axis. A linear array of 55 receivers is positioned $68 \mathrm{~cm}$ away from the center of the object with the receiver spacing $\Delta y$ of $1.5 \mathrm{~cm}$ in order to avoid any aliasing. The scattered field is uniformly sampled at receiving array $\mathrm{Rx} 1$ through $\mathrm{Rx} 55$ with frequency swept within the frequency ranges of $3-5 \mathrm{GHz}$. The general simulation procedure is identical to PEC object cases; the cylinder is rotated in clockwise direction at $9^{\circ}$ with respect to the rotational axis, that is, $(0 \mathrm{~cm}, 0 \mathrm{~cm})$, and the simulations are repeated until the object is fully rotated.

Four complete tomographic images of the dielectric cylinder based on 10 transmitted i.i.d. WGN waveforms are shown in Figures 10(a), 10(b), 10(c), and 10(d) when 1, 3, 7, and 10 noise waveforms are sequentially transmitted, respectively. Since the dielectric object is defined as a homogeneous concrete object with perfectly smooth surface, two-dimensional tomographic image of the concrete cylinder is successfully achieved.

3.5. Square Dielectric Box. Shown in Figure 11 is a bistatic noise radar system with two-dimensional scattering geometry for a square dielectric box. The length of each side of the square $(a)$ is $16 \mathrm{~cm}$, and its height is assumed to be infinitely long along the $z$-axis. Other simulation parameters, such as the position of receivers, the parameters for receiver spacing, and the rotational angle, remain unchanged. Also, complex relative permittivity and physical conditions of the object are identical to the previous simulation. Similarly, 10 i.i.d. random noise waveforms are sequentially transmitted in order to obtain the complete tomographic image of the box.

Figures 12(a), 12(b), 12(c), and 12(d) show four tomographic images of the dielectric box when 1, 3, 7, and 10 noise waveforms are transmitted, respectively. As expected, fringes are noted at the four corners of the box due to the physical shape of the target. Although the intensity of scattered field is relatively weak compared to PEC objects, the shape of the dielectric objects can be clearly inferred because the signalto-noise ratio (SNR) is infinity for all simulation cases.

\section{Implementation of UWB Noise Radar and Experimental Results}

4.1. Hardware Implementation. Figure 13 shows the block diagram of our hardware implementation of UWB noise radar in a backward scattering arrangement. The entire experiment is sequentially controlled by a desktop computer. The computer is directly connected to the electrical components: an arbitrary waveform generator (AWG) and a digital oscilloscope. The AWG is Agilent M8190A system mounted on M9602A chassis. Before the data acquisition process begins, the AWG at the transmitter side starts generating the random waveforms based on a given specification such as the number of amplitude samples, frequency bandwidth, and sampling rate. The AWG is designed to transmit the random noise waveform continuously for the duration of the measurement. Agilent DSO90804A digital oscilloscope is configured to collect and save the scattering data at the receiver side.

Also two mechanical components, that is, a turntable and a linear scanner, are controlled by the desktop computer. For the data acquisition, the target object is placed on the turntable, and a stepper motor of the turntable rotates the object a certain number of degrees. The receiving antenna is mounted on the linear scanner; the antenna moves along the linear scanner and stops at the designated coordinate to collect the data at that point. The receiving antenna is connected to Agilent Infiniium DSO90804A for collecting scattering 


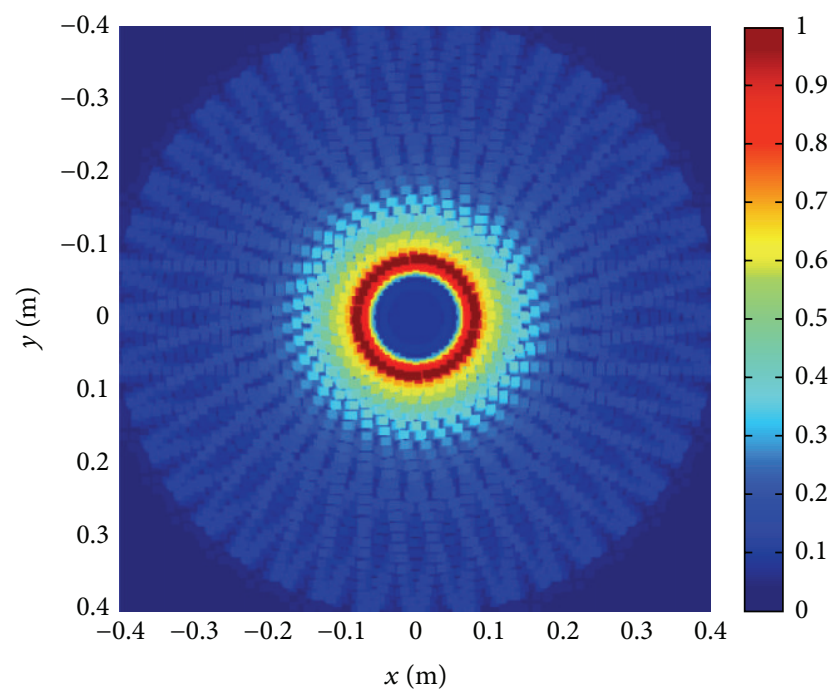

(a)

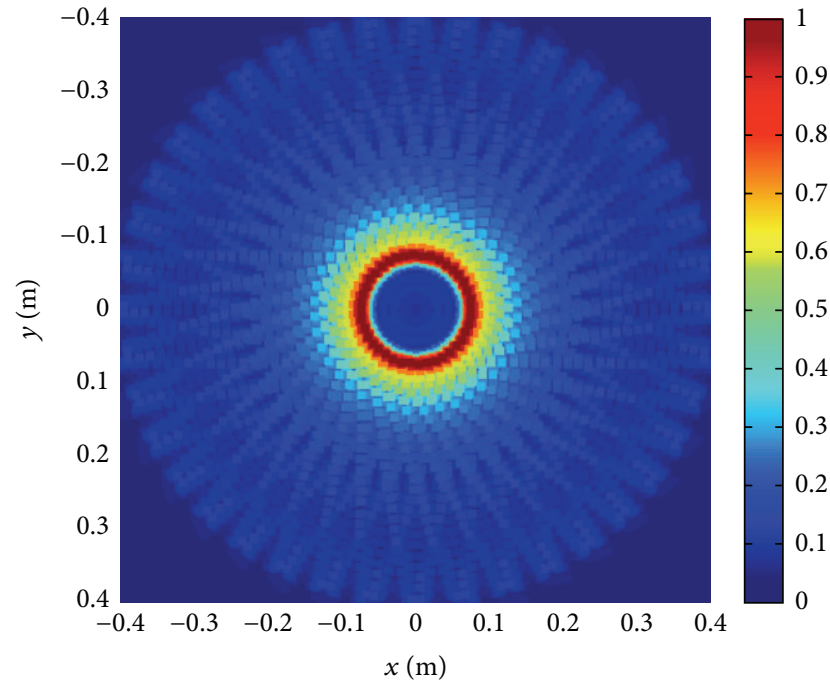

(c)

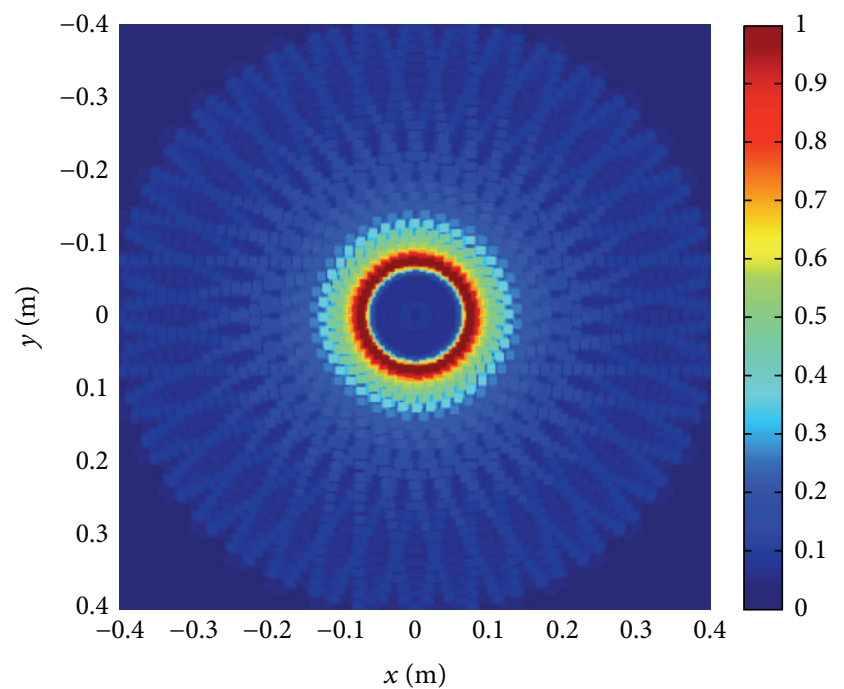

(b)

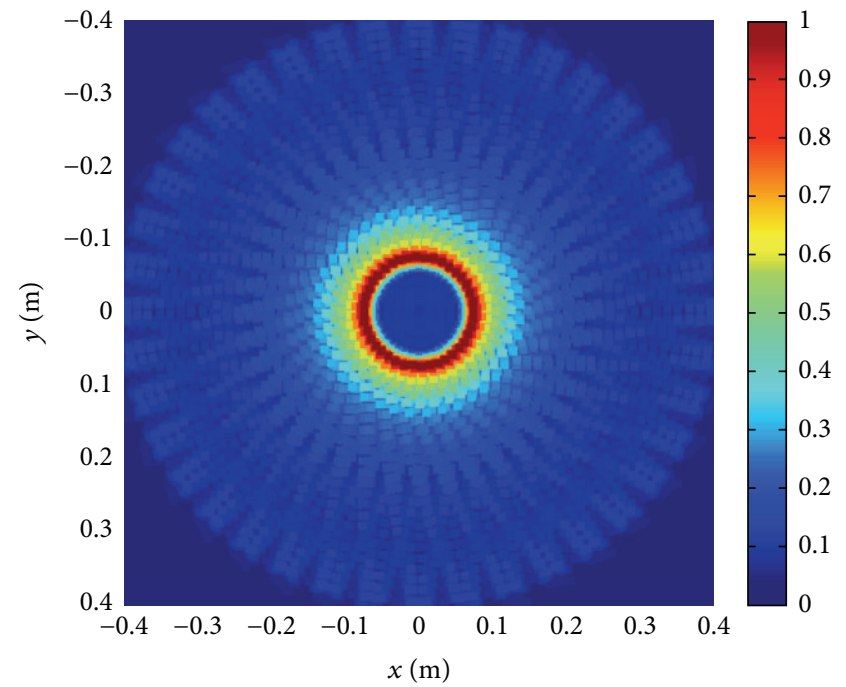

(d)

FIGURE 10: Complete tomographic image of a dielectric cylinder with a radius of $8 \mathrm{~cm}$ located at $(0 \mathrm{~cm}, 0 \mathrm{~cm})$ after summing and averaging process based on (a) one transmitted WGN waveform, (b) three transmitted WGN waveforms, (c) seven transmitted WGN waveforms, and (d) all ten transmitted WGN waveforms.

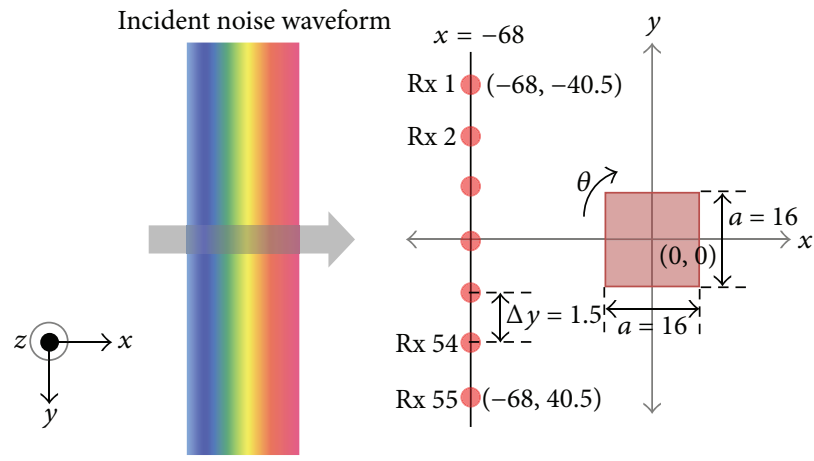

FIGURE 11: A bistatic noise radar system with two-dimensional backward scattering geometry for a square dielectric box. The target object is surrounded by vacuum. The rotational step angle is $9^{\circ}$. 


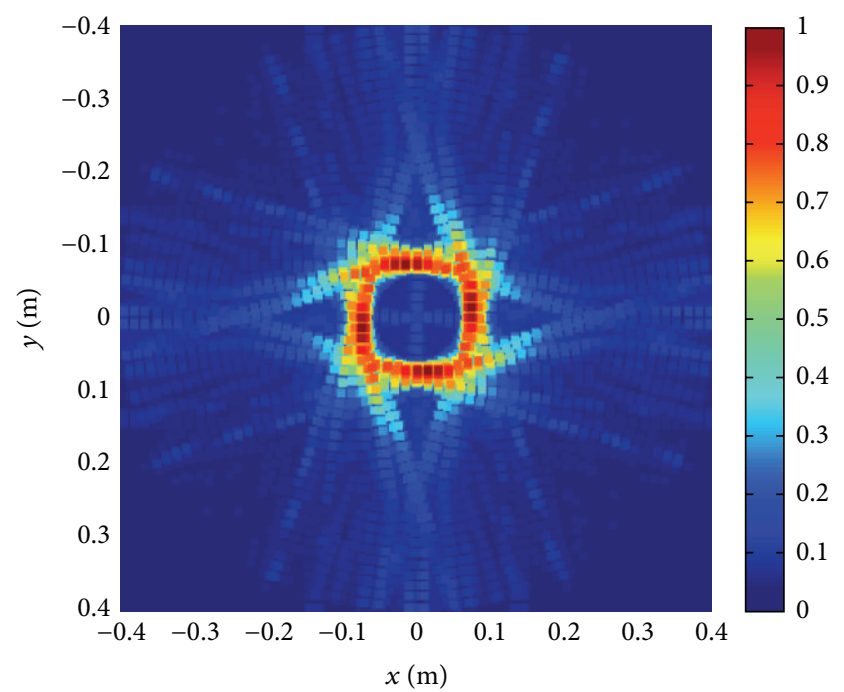

(a)

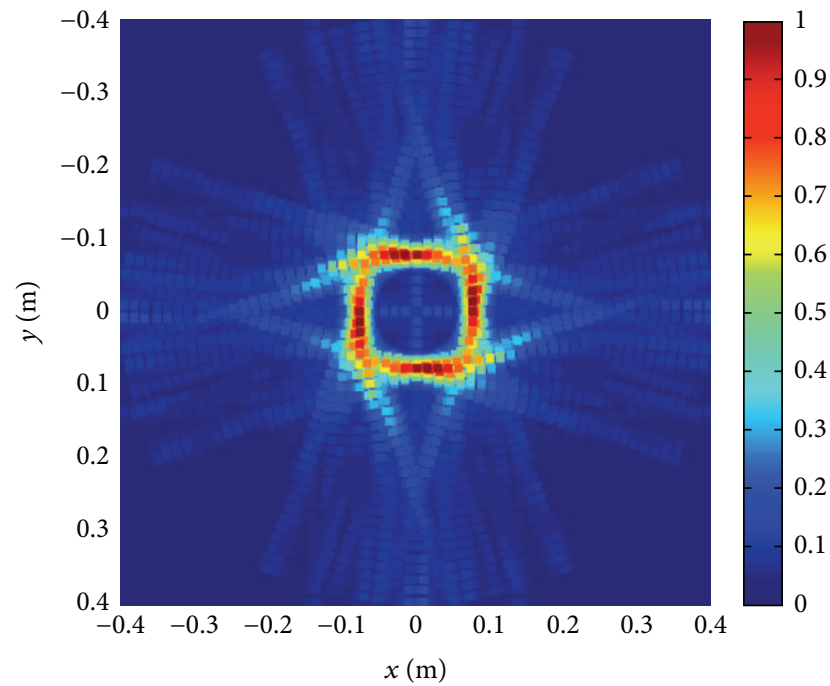

(c)

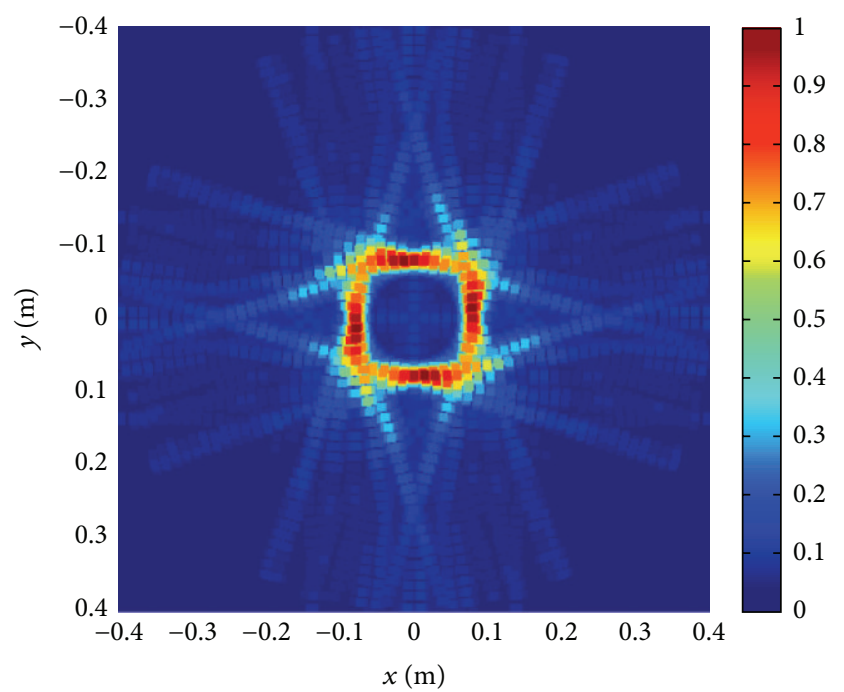

(b)

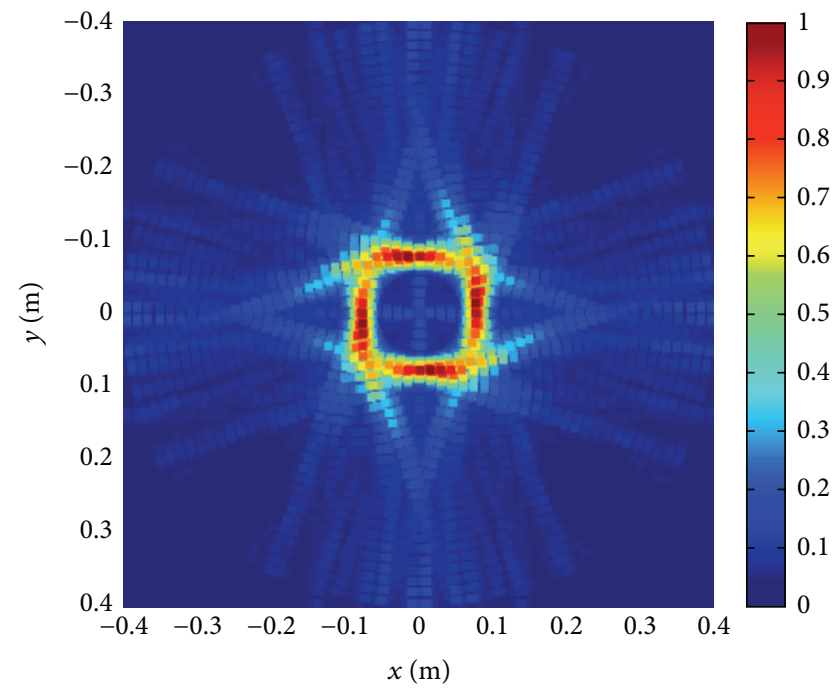

(d)

FIGURE 12: Complete tomographic image of a square PEC box with a length of $20 \mathrm{~cm}$ located at $(0 \mathrm{~cm}, 0 \mathrm{~cm})$ after summing and averaging process based on (a) one transmitted WGN waveform, (b) three transmitted WGN waveforms, (c) seven transmitted WGN waveforms, and (d) all ten transmitted WGN waveforms.

data and then sends it to the computer for tomographic image processing.

For transmitted noise waveforms, the AWG generates a bandlimited random noise waveform of $3-5 \mathrm{GHz}$ at an output power of $0 \mathrm{dBm}$. The generated noise waveform is then amplified through a Mini-Circuits ZVE-8G+ amplifier, and the measured output power of the transmitted bandlimited noise waveform for 3-5 GHz after ZVE-8G+ with $12 \mathrm{~V} \mathrm{DC}$ input bias is approximately $35 \mathrm{dBm}$.

As shown in Figure 13, the amplified noise waveform is transmitted through an A-Info dual-polarization horn antenna (LB-SJ-20180). The dual-polarized horn antenna operates over the frequency range of $2-18 \mathrm{GHz}$, and the antenna has a gain of approximately $10 \mathrm{~dB}$ over $3-5 \mathrm{GHz}$ frequency range. The position of the transmitting antenna is fixed at the center of the backward scattering geometry, but the receiving antenna is designed to move along the linear scanner to collect the scattering data. The receiving side of the system uses an identical antenna for data collection, and both transmitting and receiving antenna are installed to measure only vertically polarized scattering data.

The data collection system is controlled using a single computer. The computer is connected to four devices. The computer directly controls two Arduinos, the AWG, and the oscilloscope. Before the data collection process begins, the computer uploads the noise waveform to the AWG using a MATLAB code. The AWG continuously transmits that noise waveform for the duration of the test. One Arduino controls the position of the turntable on which the test object is placed. The second Arduino controls a linear scanner that 


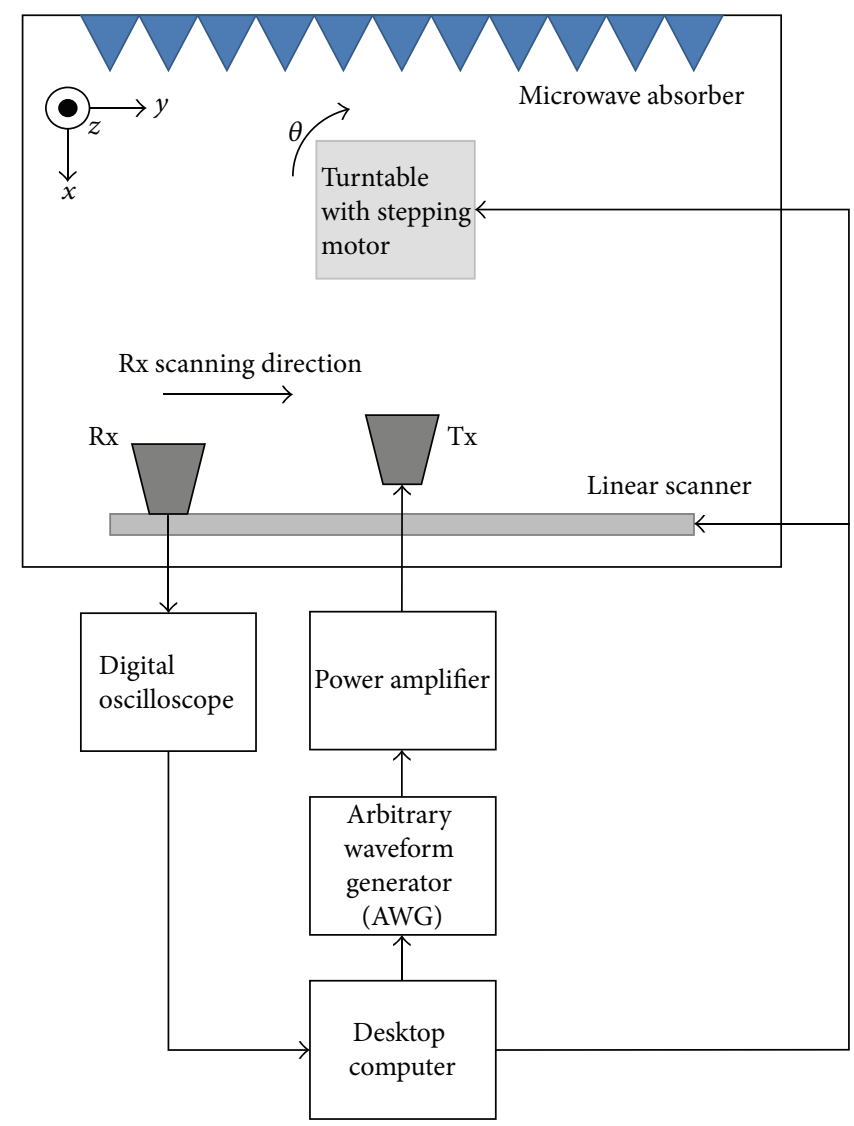

FIGURE 13: UWB noise tomography radar system block diagram.

has the receiving antenna mounted on it. The scanner stops at each point that is designated in MATLAB so data can be collected at that point. The oscilloscope collects time-domain data and then passes that data back to the computer for storage and further processing.

4.2. Data Acquisition and Image Processing. Figure 14 shows a picture of actual experimental hardware implementation. The transmitted noise waveforms are not identical between each measurement; however, the i.i.d. white Gaussian noise waveforms are generated and transmitted for the experiments. Each time the linear scanner stops, DSO90804A collects a total of 363,000 amplitude samples. As previously described, the turntable rotates in steps of $9^{\circ}$ after a single line scan has been completed, and the data acquisition process repeats until the object has fully rotated for $360^{\circ}$. The total data stored of a single target object with complete $360^{\circ}$ rotation are in $40 \times$ 363,000 matrix array for tomographic image processing at a later point in time.

The spectral response of the transmitted random noise waveform is directly related to the image quality. If the insufficient amount of scattering data is collected, an unrecognizable image of the target may be formed due to the fluctuation of the spectrum response. Although a long sample length or the averaging of multiple samples is necessary to achieve a flat frequency spectrum, the data size of $40 \times 363,000$ matrix array may not be processed at the same time due to the computational limitations of the desktop computer. For the computational efficiency, each of the 363,000 point samples is split into $N$ segments for processing and then summed and averaged to create an image. A total of $N$ images are reconstructed for each rotational angle where the turntable stops, and the final tomographic image is generated via sum and average of $N$ images. After measurement with complete $360^{\circ}$ rotations, a total of $40 \times N$ final tomographic images are created. Using image processing, these $40 \times N$ images are combined into one complete tomographic image [52]. For this paper, all collected data samples are split into 200 segments such that the complete tomographic image of each target object shown in this chapter is reconstructed based on $40 \times 200$ images. The general experiment process is identical to Figure 2.

Both metallic and dielectric cylinders with different shapes are measured for tomographic image reconstructions. The metallic objects are constructed from sheets of aluminum. For the dielectric target objects, they are concrete blocks. The test objects are placed on the center of the turntable one at a time. Table 2 shows the actual target objects for which the tomographic images are presented herein and compared with the simulation results.

4.3. Circular Metallic Cylinder. Top view and side view of experiment configuration for a circular metallic cylinder are shown in Figure 15. The measured radius $(r)$ and height $(h)$ 
TABLE 2: Target objects imaged by the tomographic noise radar.

\begin{tabular}{lcc}
\hline Object material & Object description & Dimension \\
\hline \multirow{3}{*}{ Metal } & Circular cylinder & Square box \\
& Triangular prism & $20.3 \mathrm{~cm}$ diameter $\times 40.6 \mathrm{~cm}$ height $\left(8^{\prime \prime}\right.$ diameter $\times 16^{\prime \prime}$ height $)$ \\
\hline \multirow{3}{*}{ Concrete } & Circular cylinder & $20.3 \mathrm{~cm} \times 20.3 \mathrm{~cm} \times 61 \mathrm{~cm}$ height $\left(8^{\prime \prime} \times 8^{\prime \prime} \times 24^{\prime \prime}\right)$ \\
& Square block & $20.3 \mathrm{~cm} \mathrm{side} \times 30.5 \mathrm{~cm}$ height $\left(8^{\prime \prime}\right.$ side $\times 12^{\prime \prime}$ height $)$ \\
\hline
\end{tabular}
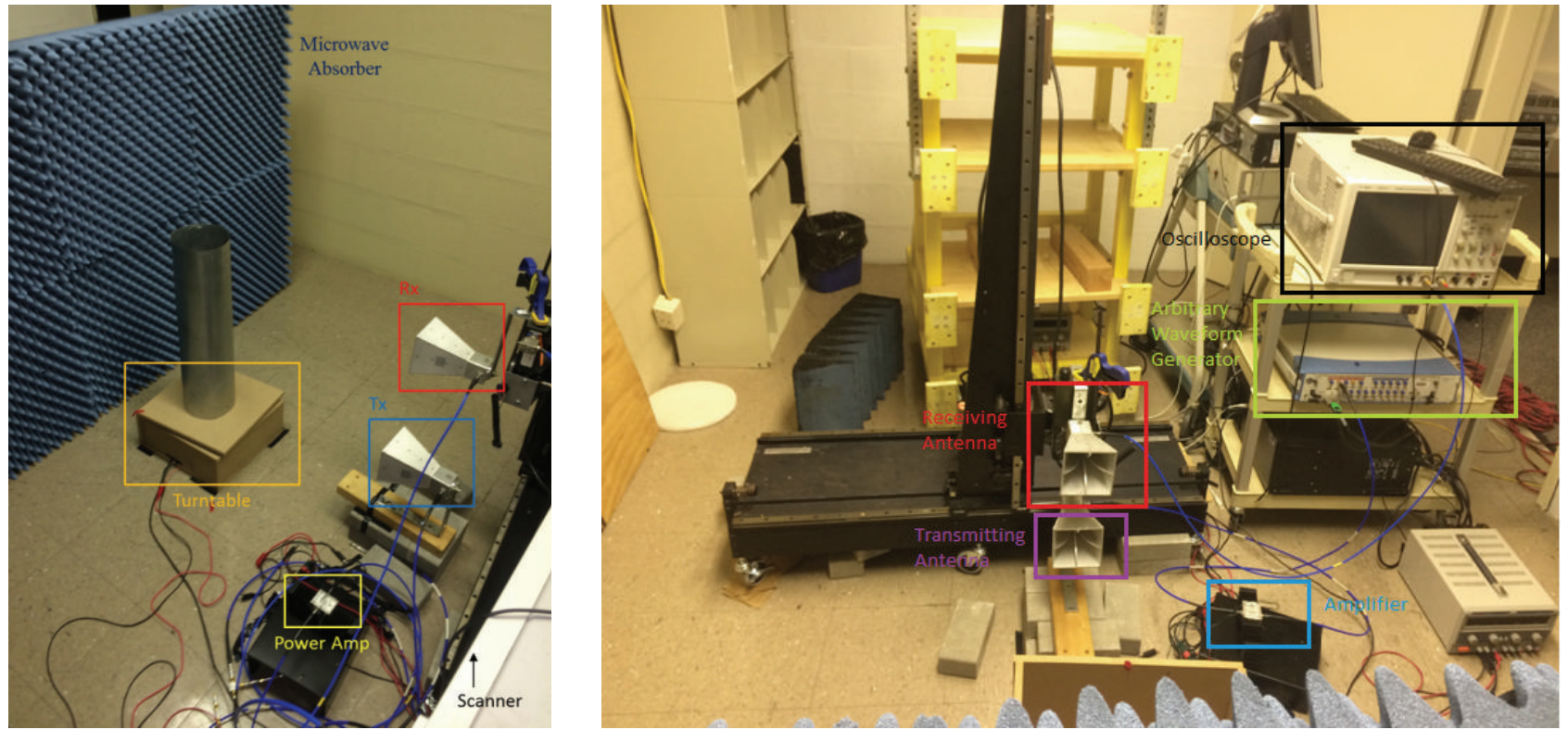

FIGURE 14: A metal cylinder placed on the turntable. Also shown are the transmitting and receiving antenna and microwave absorber behind the target to minimize reflections from the back, as well as the test equipment and the scanner [52].
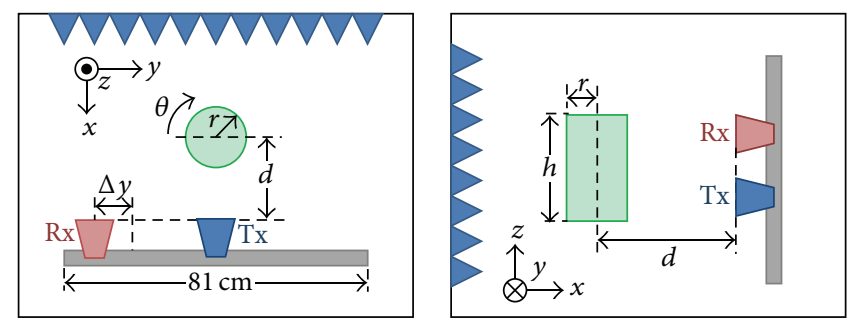

FIGURE 15: Top and side view of experiment configuration for a circular metallic cylinder. The cylinder is placed on the turntable for rotation. Also the microwave absorber behind the target is shown to minimize reflections from the back. The rotational step angle is $9^{\circ}$.

of the cylinder are approximately $10.2 \mathrm{~cm}$ and $40.6 \mathrm{~cm}$, respectively, and the measured distance $(d)$ from the center of the cylinder to the edge of a transmitting antenna is approximately $66 \mathrm{~cm}$. A complete view of the circular metallic cylinder is shown in Figure 16. The transmitting antenna (Tx) is fixed at the center of the object, and the receiving antenna $(\mathrm{Rx})$ is moving along the $y$-axis to collect the scattering data. Moving interval $(\Delta y)$ for $\mathrm{Rx}$ is $1.5 \mathrm{~cm}$; that is, scattering data are measured and recorded for 55 different positions along $y$-axis from $(-66 \mathrm{~cm},-40.5 \mathrm{~cm})$ and $(-66 \mathrm{~cm}, 40.5 \mathrm{~cm})$.
Figure 17 shows the final tomographic image of the circular metallic cylinder based on the measured scattering data. The tomographic image of the cylinder is correctly reconstructed; however, the image quality is not as good when compared to the simulated images displayed in Figure 4. Since the experiment is performed in the room surrounded by concrete walls, the image is contaminated by the additional scattering due to concrete walls where no microwave absorbers are present. Also, unwanted noise from active RF components and equipment degrades the image quality. 

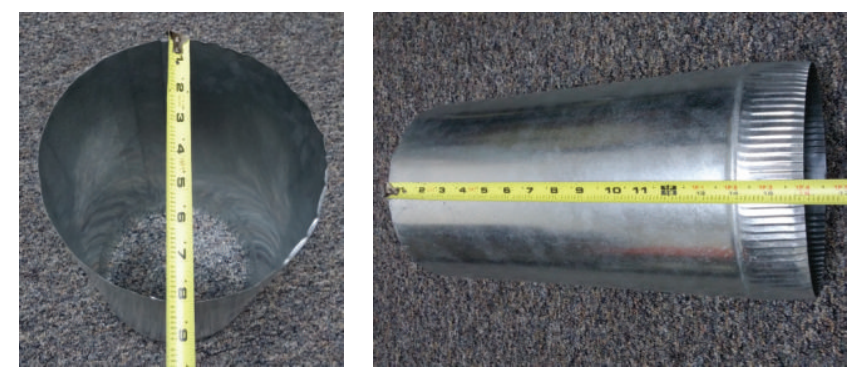

Figure 16: The metallic cylinder used for the experiment. Measured dimensions are in inches.

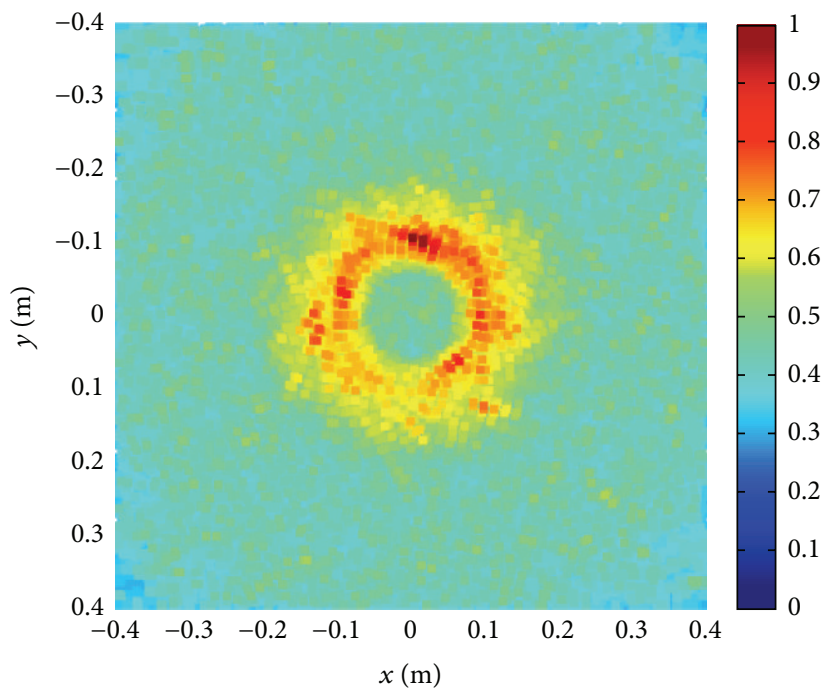

FIGURE 17: Tomographic image of the metallic cylinder based on the measured data.

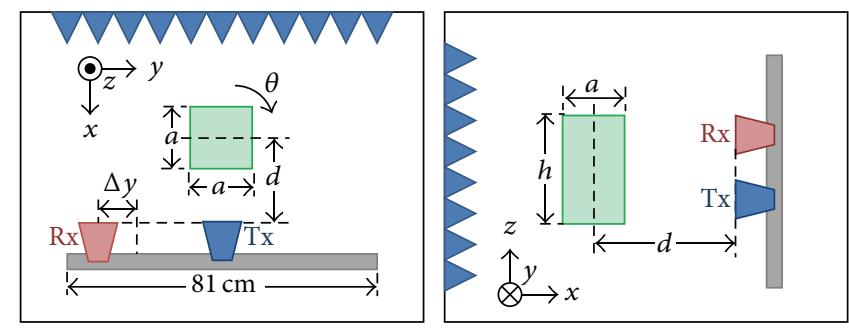

Figure 18: Top and side view of experiment configuration for a square metallic box. The box is placed on the turntable for rotation. The rotational step angle is $9^{\circ}$.

However, despite these unwanted reflections, the cylindrical shape is well recognized.

4.4. Square Metallic Box. Top view and side view of experiment configuration for a square metallic cylinder are shown in Figure 18. The dimension of the box is $20.3 \times 20.3 \times 61 \mathrm{~cm}$, and the measured distance $(d)$ from the center of the cylinder to the edge of a transmitting antenna is approximately $66 \mathrm{~cm}$. Other experimental configuration remains unchanged. A complete view of the square metallic box is shown in Figure 19.

As shown in Figure 20, the tomographic image of the box is correctly reconstructed, and the shape can be recognized.
Similarly, fringes are imaged due to the sharp corners of the box. As before, the image quality is degraded by the additional scattering data due to concrete walls and unwanted noise from active RF components.

4.5. Equilateral Triangular Metallic Prism. Figure 21 shows top view and side view of experiment configuration for an equilateral triangular metallic prism. The length of all sides of the triangle is $20.3 \mathrm{~cm}$, and the height $(h)$ of the prism is $30.5 \mathrm{~cm}$. The measured distance $(d)$ from the center of the prism to the edge of a transmitting antenna is approximately $66 \mathrm{~cm}$. Figures 22 and 23 depict the actual triangular prism and the complete tomographic image with a full $360^{\circ}$ rotation 

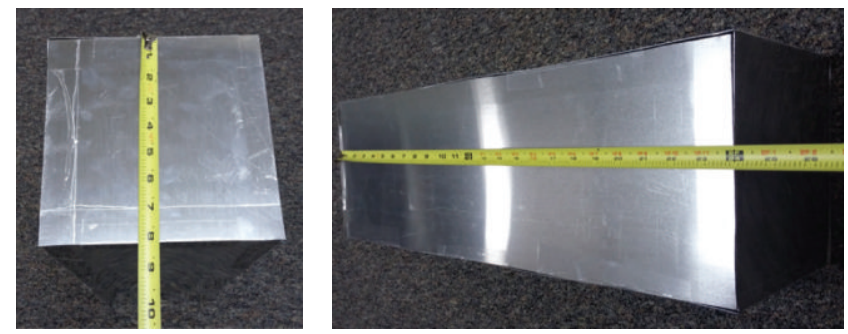

FIgURE 19: The metallic square box used for the experiment. Measured dimensions are in inches.

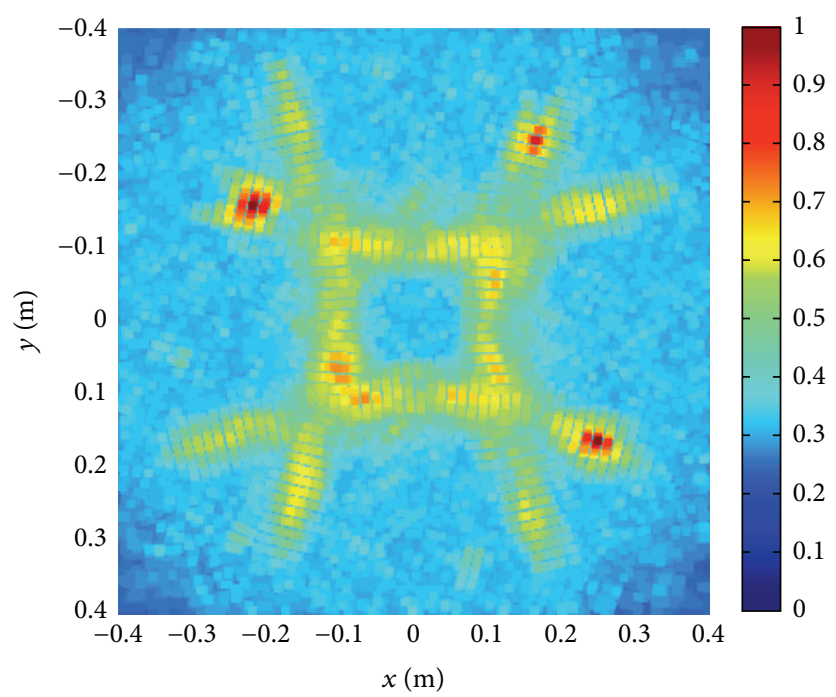

FIGURE 20: Tomographic image of the metallic box based on the measured data.

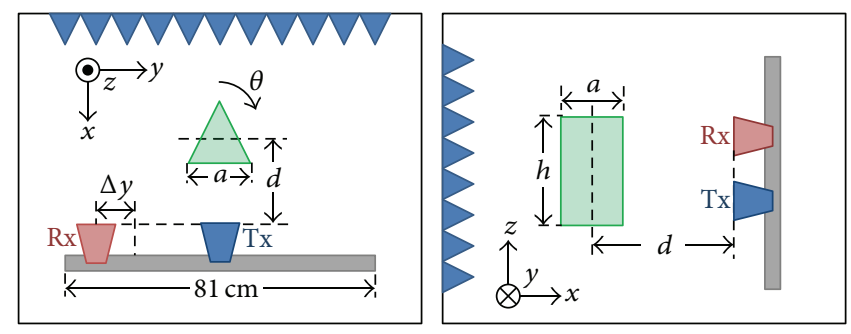

FIGURE 21: Top and side view of experiment configuration for an equilateral triangular metallic prism. The rotational step angle is $9^{\circ}$.
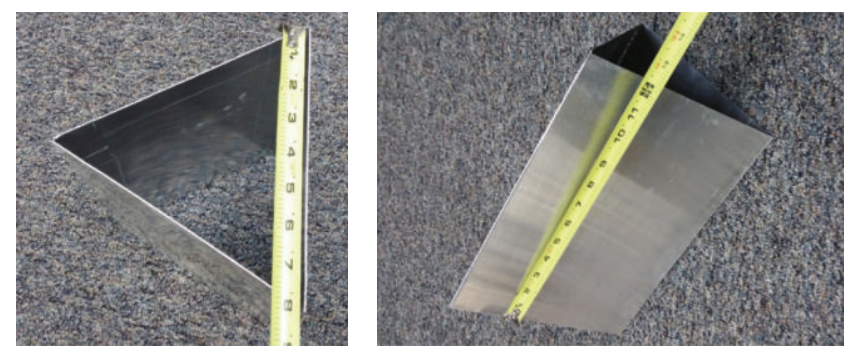

FIGURE 22: The metallic prism used for the experiment. Measured dimensions are in inches. 


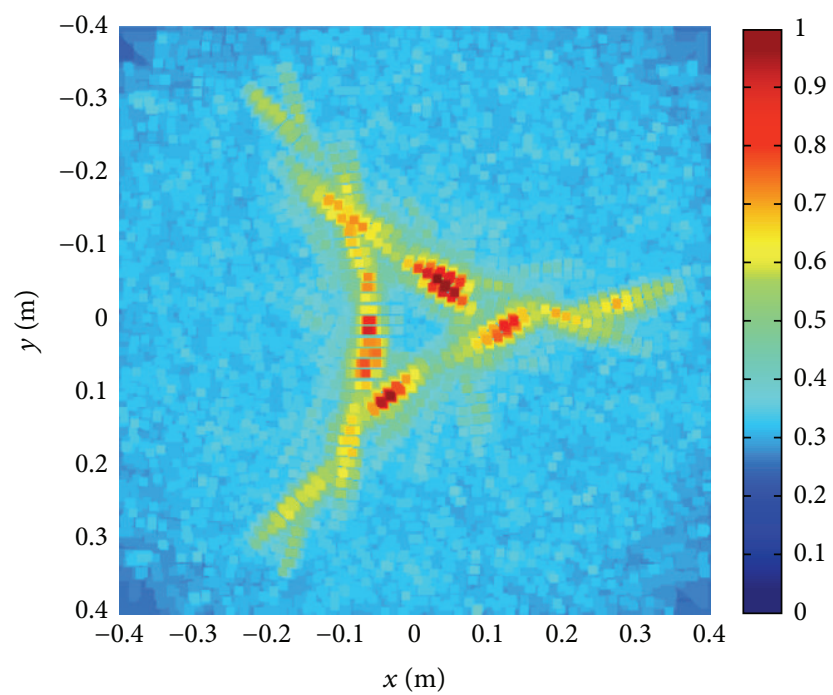

FIGURE 23: Tomographic image of the metallic prism based on the measured data.

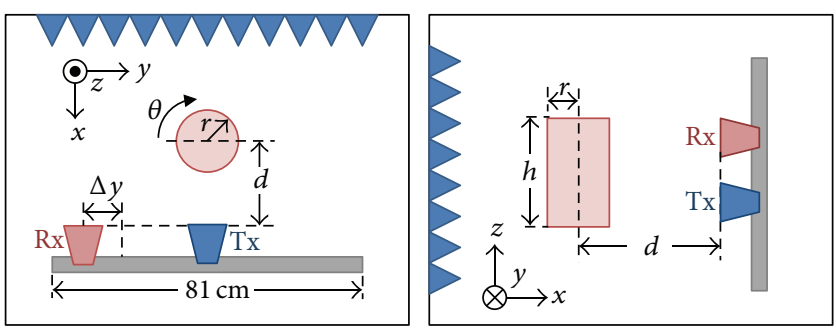

FIGURE 24: Top and side view of experiment configuration for a circular concrete cylinder. The rotational step angle is $9^{\circ}$.

of the object, respectively. The cross-section image of the prism can be clearly inferred in Figure 23.

4.6. Circular Concrete Cylinder. Figure 24 shows top view and side view of experiment configuration for a circular concrete cylinder. The measured radius $(r)$ and height $(h)$ of the cylinder are approximately $7.6 \mathrm{~cm}$ and $30.5 \mathrm{~cm}$, respectively, and the measured distance $(d)$ from the center of the cylinder to the edge of a transmitting antenna is approximately $66 \mathrm{~cm}$. A view of the concrete cylinder is shown in Figure 25. For the concrete cylinder measurement, moving interval $(\Delta y)$ for $\mathrm{Rx}$ is $3 \mathrm{~cm}$; that is, scattering data are measured and recorded for 29 different positions along $y$-axis from $(-66 \mathrm{~cm},-40.5 \mathrm{~cm})$ and $(-66 \mathrm{~cm}, 40.5 \mathrm{~cm})$.

Figure 26 shows the complete tomographic image of the circular concrete cylinder based on the measured scattering data. Although the tomographic image of the dielectric cylinder is successfully obtained, the image is not truly vivid compared to the metallic circular cylinder, as expected. The measured scattering field intensity of concrete object is not as strong as the metallic object case. Also the surface roughness and the composition of concrete cylinder affect the scattering behavior, which degrades the image quality compared to the metallic cylinder case.

4.7. Square Concrete Block. Top view and side view of experiment configuration for a square concrete block are shown in Figure 27. The dimension of the block is $15.2 \times$ $15.2 \times 28 \mathrm{~cm}$. The measured distance $(d)$ from the center of the cylinder to the edge of a transmitting antenna is approximately $66 \mathrm{~cm}$. For the concrete block measurement, the transmitting antenna ( $\mathrm{Tx})$ is fixed at the center of the object, and only receiving antenna $(\mathrm{Rx})$ is moving along the $y$-axis to collect the scattering data for 29 different scanning points; that is, $\Delta y=3 \mathrm{~cm}$, from $(-66 \mathrm{~cm},-40.5 \mathrm{~cm})$ and $(-66 \mathrm{~cm}, 40.5 \mathrm{~cm})$. The turntable is configured to rotate $9^{\circ}$ after a single line scan, and the scattering data is measured for 40 different angles. Figures 28 and 29 show the actual concrete block and the complete tomographic image with a full $360^{\circ}$ rotation of the object, respectively. The image of the concrete block is affected by the measured field intensity. Since the surface of the concrete block is rough, the image quality is not comparable to metallic box case. However, the experimental results are in good agreement with the simulation results shown in the previous section.

4.8. Semicircular Concrete Cylinder. Top view and side view of experiment configuration for a semicircular concrete cylinder are shown in Figure 30. The measured radius $(r)$ and height $(h)$ of the cylinder are approximately $7.6 \mathrm{~cm}$ and $30.5 \mathrm{~cm}$, respectively, and the measured distance $(d)$ from the center of the cylinder to the edge of a transmitting antenna is approximately $66 \mathrm{~cm}$. A view of the concrete cylinder is 

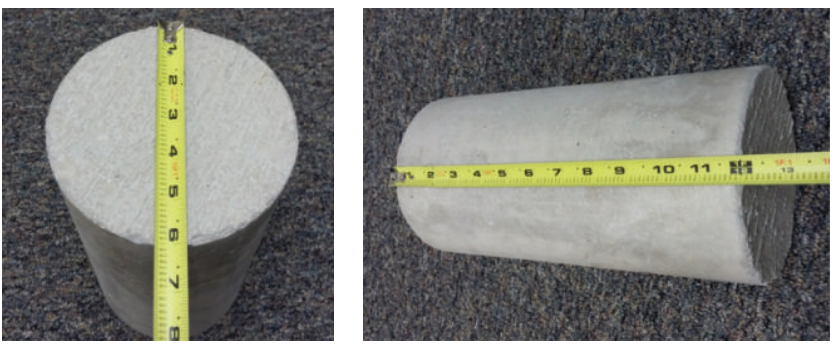

FIgURE 25: The concrete cylinder used for the experiment. Measured dimensions are in inches.

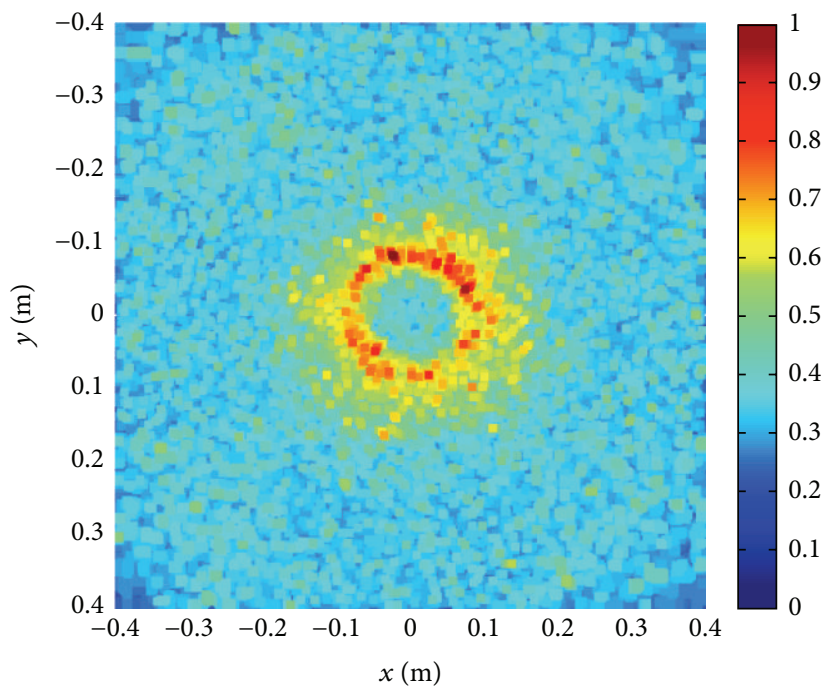

FIGURE 26: Tomographic image of the concrete cylinder based on the measured data.

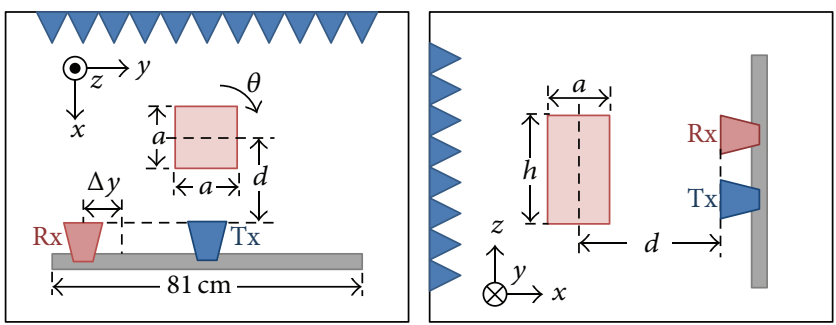

FIgURE 27: Top and side view of experiment configuration for a square concrete block. The rotational step angle is $9^{\circ}$.
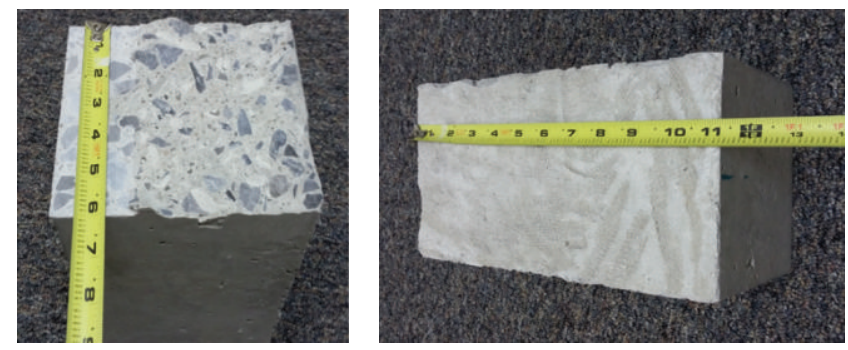

FIGURE 28: The concrete block used for the experiment. Measured dimensions are in inches. 


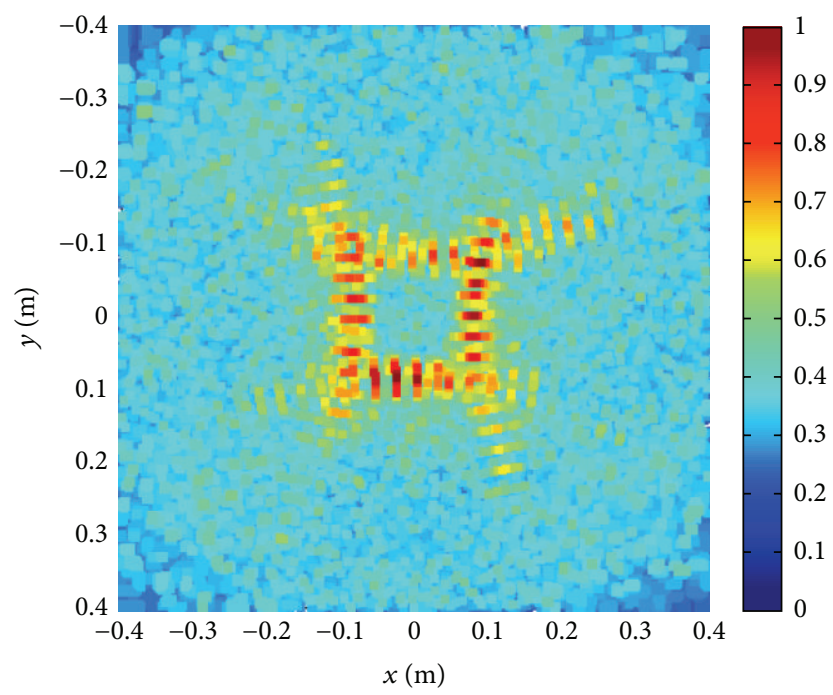

FIGURE 29: Tomographic image of the concrete block based on the measured data.

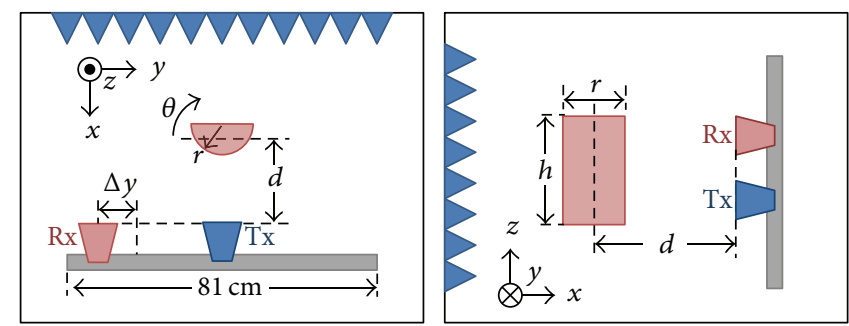

Figure 30: Top and side view of experiment configuration for a semicircular concrete cylinder. The rotational step angle is $9^{\circ}$.

shown in Figure 31. For the semicircular concrete cylinder measurement, moving interval $(\Delta y)$ for $\mathrm{Rx}$ is changed to $1.5 \mathrm{~cm}$; that is, scattering data are measured and recorded for 55 different positions along $y$-axis from $(-66 \mathrm{~cm},-40.5 \mathrm{~cm})$ and $(-66 \mathrm{~cm}, 40.5 \mathrm{~cm})$. As seen in Figure 31, the surface of the semicircular concrete cylinder is not perfectly smooth and homogeneous.

Figure 32 shows the complete tomographic image of the semicircular concrete cylinder based on the measured scattering data. Since the object has two sharp edges at the back side, the fringes are observed around the corners of the flat side. The surface roughness and the composition of concrete cylinder definitely degrade the image quality, which is similar to the previous concrete object cases.

4.9. Imaging through Barriers. A few of the targets were placed within optically opaque containers, such as a cardboard box and a wooden box, and tomographic images were produced.

The size of the cardboard box was $50.8 \mathrm{~cm} \times 40.6 \mathrm{~cm} \times$ $54.1 \mathrm{~cm}$ and its wall thickness was $4 \mathrm{~mm}$. Figure 33 shows the complete tomographic images of the circular metallic cylinder (Figure 16) and the equilateral triangular metallic prism (Figure 22). By comparing Figures 33(a) and 17 and also Figures 33(b) and 23, it can be observed that while the cardboard adds a small amount of noise, the target object is still clearly visible and discernible.

Objects were also concealed within a wooden box of size $38.1 \mathrm{~cm} \times 38.1 \mathrm{~cm} \times 38.1 \mathrm{~cm}$ and $1.27 \mathrm{~cm}$ wall thickness. Figure 34 shows the complete tomographic images of the circular metallic cylinder (Figure 16), the square metallic box (Figure 19), the circular concrete cylinder (Figure 25), and the square concrete block (Figure 28). The wooden box adds more noise to the images when compared to the corresponding images obtained in the free space environment, but the target objects are still visible and their shapes recognizable. Objects having a square cross section are imaged well, while images of objects with a circular cross section are somewhat distorted.

\section{Conclusions}

Radar tomography using UWB random noise waveforms is accomplished while maintaining the advantages of UWB noise radar such as LPI and high-resolution imaging capability. Theoretical analysis, numerical simulation results, and hardware implementation of the bistatic UWB noise tomography radar are presented in this paper. We conclude that the tomographic images of various target scenarios are correctly 

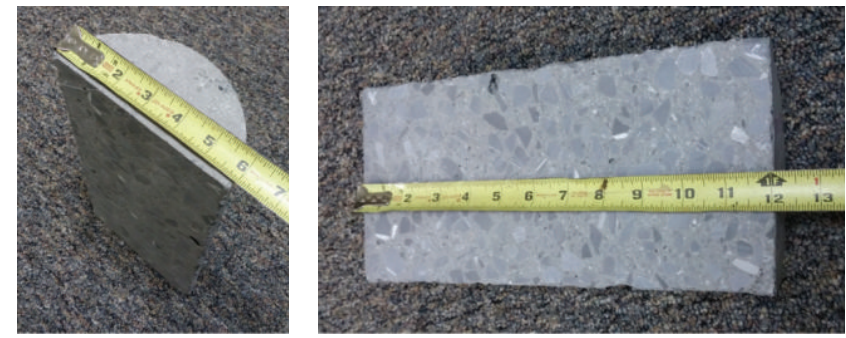

FIGURE 31: The semicircular concrete cylinder used for the experiment. Measured dimensions are in inches.

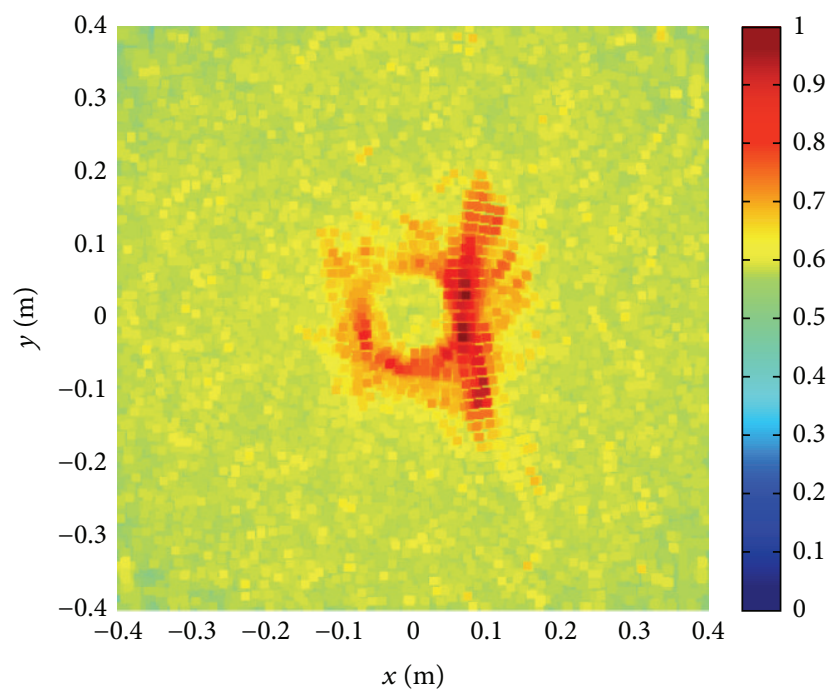

FIGURE 32: Tomographic image of the semicircular concrete cylinder based on the measured data.

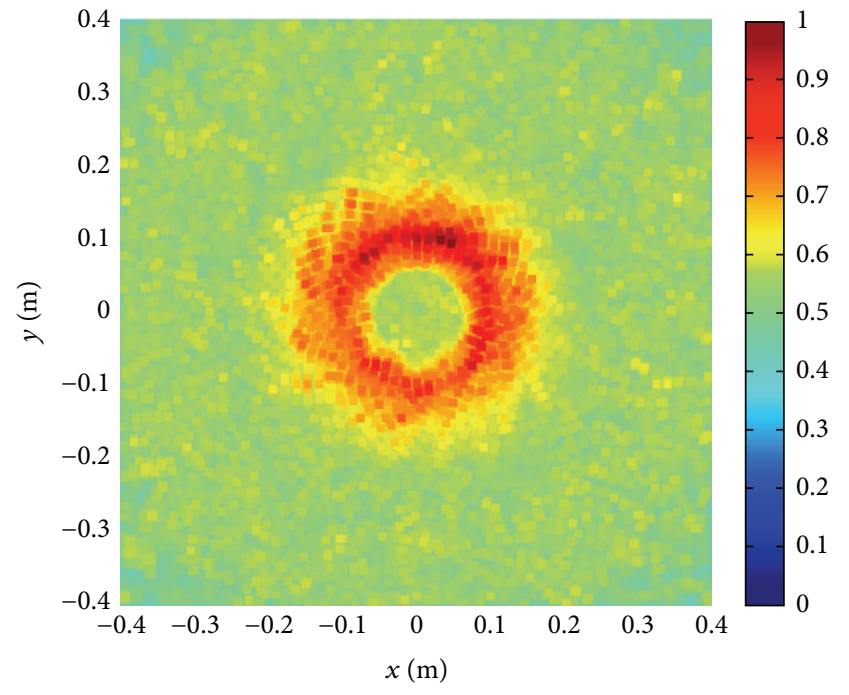

(a)

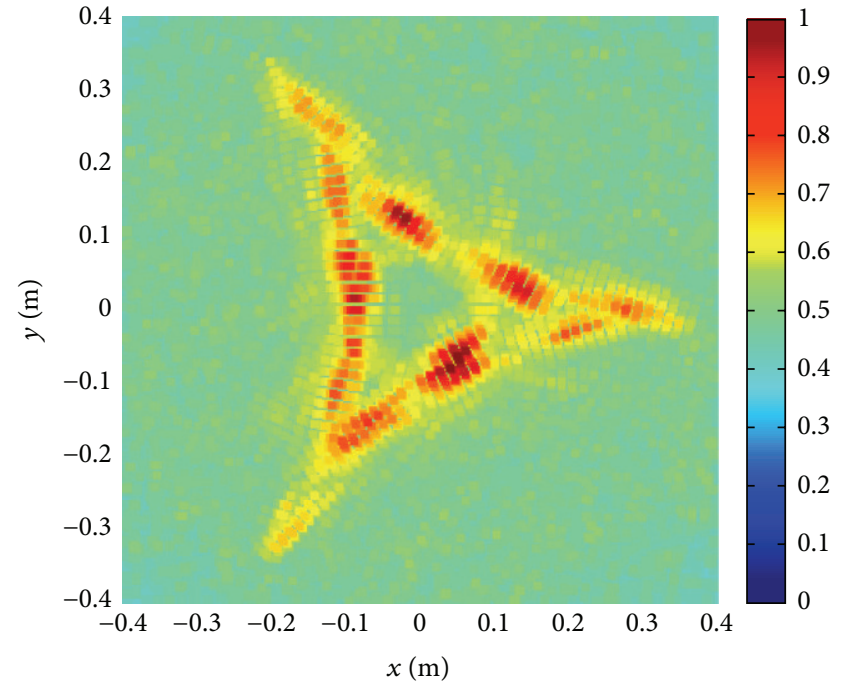

(b)

FIGURE 33: Tomographic images of (a) the circular metallic cylinder and (b) equilateral triangular metallic prism concealed within a cardboard box based on the measured data. 


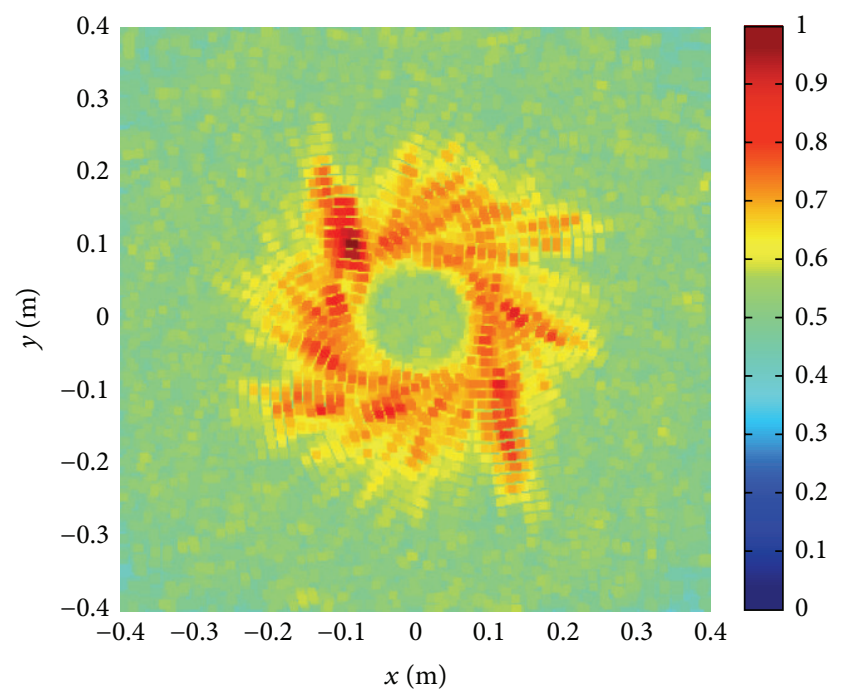

(a)

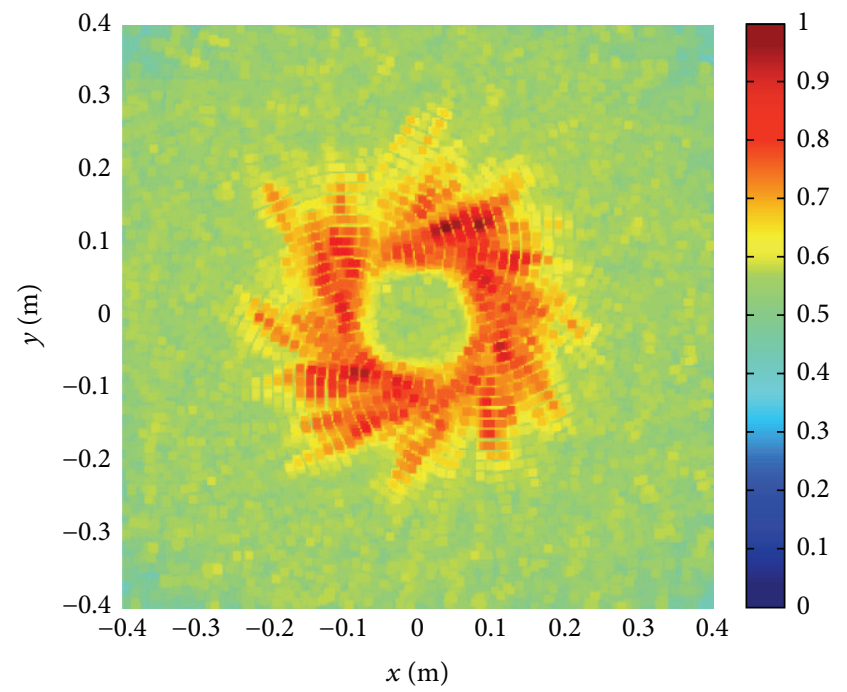

(c)

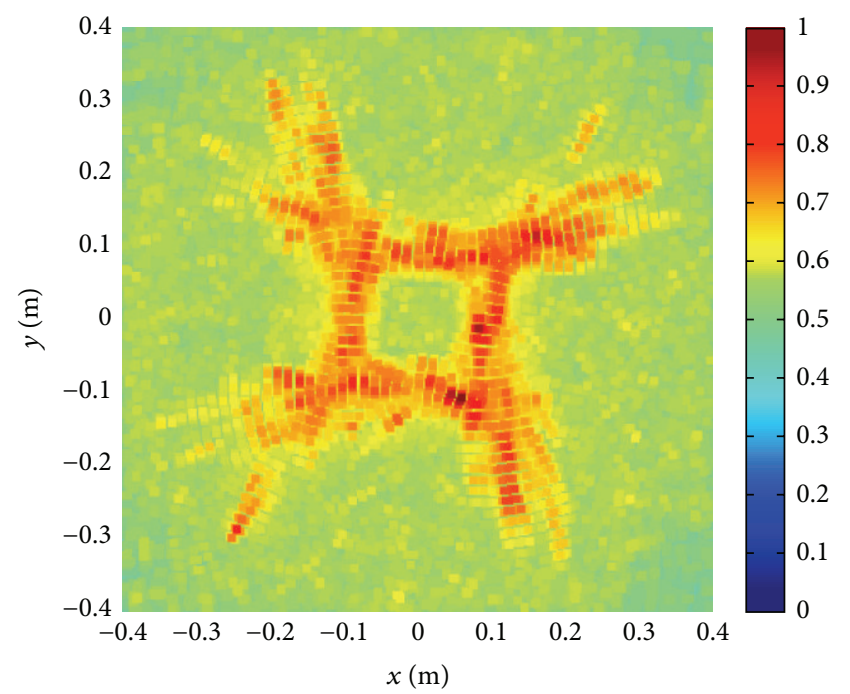

(b)

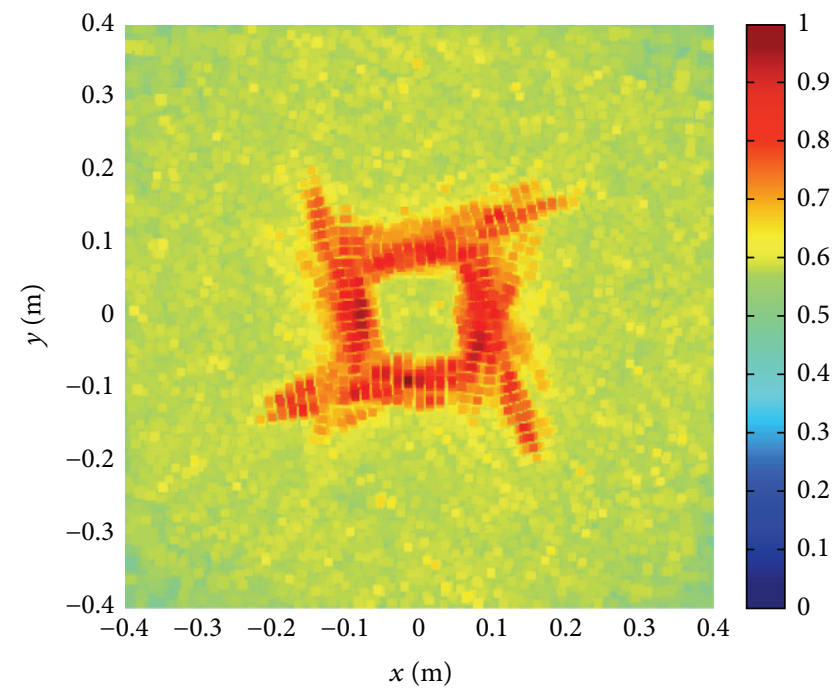

(d)

FIgURE 34: Tomographic images of (a) the circular metallic cylinder, (b) the square metallic box, (c) the circular concrete cylinder, and (d) the square concrete block concealed within a wooden box based on the measured data.

formed with UWB random noise waveforms, showing a good agreement between simulation and experiment results for both metallic and dielectric target object cases. Based on the comparison of simulation and experimental results, the presence of unwanted noise truly degrades the image quality; however, the suppression of unwanted noise contributions by controlling SNR can help to enhance the image quality of tomographic images in practical radar imaging.

\section{Competing Interests}

The authors declare that they have no competing interests.

\section{Acknowledgments}

This work was supported by the Air Force Office of Scientific Research (AFOSR) Contract no. FA9550-12-1-0164.

\section{References}

[1] E. K. Walton, "Use of fixed-range noise radar for moving-vehicle identification," in Proceedings of the ARL (Army Research Laboratory) Symposium on Sensors and Electron Devices Symposium, College Park, Md, USA, January 1997.

[2] H. Sun, Y. Lu, and G. Liu, "Ultra-wideband technology and random signal radar: an ideal combination," IEEE Aerospace and Electronic Systems Magazine, vol. 18, no. 11, pp. 3-7, 2003.

[3] R. M. Narayanan and X. Xu, "Principles and applications of coherent random noise radar technology," in Noise in Devices and Circuits, vol. 5113 of Proceedings of SPIE, pp. 503-514, Santa Fe, NM, USA, June 2003.

[4] G. Liu, X. Shi, and J. Lu, "Random FM-CW radar and its ECCM," in Proceedings of the Record of the Chinese Institute of Electronics International Conference on Radar (CIE ICR '86), pp. 155-160, Nanjing, China, November 1986. 
[5] N. Gonget, P. Y. Arques, and L. Martinet, "Surveillance radar waveform fitted for antistealthness and for countercountermeasures: evaluation," in Proceedings of the 8th European Signal Processing Conference (EUSIPCO '96), Paper no. PSO.10, Trieste, Italy, September 1996.

[6] D. S. Garmatyuk and R. M. Narayanan, "ECCM capabilities of an ultrawideband bandlimited random noise imaging radar," IEEE Transactions on Aerospace and Electronic Systems, vol. 38, no. 4, pp. 1243-1255, 2002.

[7] J. R. Forrest and J. P. Meeson, "Solid-state microwave noise radar," Electronics Letters, vol. 12, no. 15, pp. 365-366, 1976.

[8] L. Guosui, G. U. Hong, and S. U. Weimin, “The development of random signal radars," IEEE Transactions on Aerospace and Electronic Systems, vol. 35, no. 3, pp. 770-777, 1999.

[9] J. Sachs, M. Kmec, H. C. Fritsch et al., "Ultra-wideband pseudonoise sensors," Applied Radio Electronics, vol. 12, no. 1, pp. 79-88, 2013.

[10] X. Xu and R. M. Narayanan, "FOPEN SAR imaging using UWB step-frequency and random noise waveforms," IEEE Transactions on Aerospace and Electronic Systems, vol. 37, no. 4, pp. 1287-1300, 2001.

[11] R. M. Narayanan, X. Xu, and J. A. Henning, "Radar penetration imaging using ultra-wideband (UWB) random noise waveforms," IEE Proceedings-Radar, Sonar and Navigation, vol. 151, no. 3, pp. 143-148, 2004.

[12] K. Lukin and V. Konovalov, "Through wall detection and recognition of human beings using noise radar sensors," in Proceedings of the NATO RTO SET Symposium on Target Identification and Recognition Using RF Systems, pp. P15.1P15.12, Oslo, Norway, October 2004.

[13] J. Sachs, M. Aftanas, S. Crabbe et al., "Detection and tracking of moving or trapped people hidden by obstacles using ultrawideband pseudo-noise radar," in Proceedings of the 5th European Radar Conference, pp. 408-411, IEEE, Amsterdam, The Netherlands, October 2008.

[14] R. M. Narayanan, "Through-wall radar imaging using UWB noise waveforms," Journal of the Franklin Institute, vol. 345, no. 6, pp. 659-678, 2008.

[15] E. K. Walton, "Ground penetrating radar using ultra-wideband noise," in Proceedings of the Second Government Workshop on Ground Penetrating Radar (GPR '93), p. 121, Columbus, Ohio, USA, October 1993.

[16] E. K. Walton and L. Cai, "Signatures of surrogate mines using noise radar," in Detection and Remediation Technologies for Mines and Minelike Targets III, vol. 3392 of Proceedings of SPIE, pp. 615-626, Orlando, Fla, USA, September 1998.

[17] C. K. Chan and N. H. Farhat, "Frequency swept tomographic imaging of three-dimensional perfectly conducting objects," IEEE Transactions on Antennas and Propagation, vol. 29, no. 2, pp. 312-319, 1981.

[18] K. K. Knaell and G. P. Cardillo, "Radar tomography for the generation of three-dimensional images," IEE Proceedings: Radar, Sonar and Navigation, vol. 142, no. 2, pp. 54-60, 1995.

[19] L. Jofre, A. Broquetas, J. Romeu et al., "UWB tomographic radar imaging of penetrable and impenetrable objects," Proceedings of the IEEE, vol. 97, no. 2, pp. 451-464, 2009.

[20] W. C. Chew, W. H. Weedon, and M. Moghaddam, "Inverse scattering and imaging using broadband time-domain data," in Ultra-Wideband, Short-Pulse Electromagnetics 2, L. Carin and L. B. Felsen, Eds., vol. 2, pp. 549-562, Springer, New York, NY, USA, 1995.
[21] E. Porter, A. Santorelli, M. Coates, and M. Popović, "An experimental system for time-domain microwave breast imaging," in Proceedings of the 5th European Conference on Antennas and Propagation (EUCAP '11), pp. 2906-2910, IEEE, Rome, Italy, April 2011.

[22] H. J. Shin, R. M. Narayanan, and M. Rangaswamy, "Tomographic imaging with ultra-wideband noise radar using timedomain data," in Radar Sensor Technology XVII, vol. 8714 of Proceedings of SPIE, pp. 1-9, Baltimore, Md, USA, May 2013.

[23] A. C. Kak, "Computerized tomography with X-ray, emission, and ultrasound sources," Proceedings of the IEEE, vol. 67, no. 9, pp. 1245-1272, 1979.

[24] L. Garnero, A. Franchois, J.-P. Hugonin, C. Pichot, and N. Joachimowicz, "Microwave imaging-complex permittivity reconstruction by simulated annealing," IEEE Transactions on Microwave Theory and Techniques, vol. 39, no. 11, pp. 1801-1807, 1991.

[25] S. Valle, L. Zanzi, and F. Rocca, "Radar tomography for NDT: comparison of techniques," Journal of Applied Geophysics, vol. 41, no. 2-3, pp. 259-269, 1999.

[26] R. Ciocan and H. B. Jiang, "Model-based microwave image reconstruction: simulations and experiments," Medical Physics, vol. 31, no. 12, pp. 3231-3241, 2004.

[27] L. Huang and Y. Lu, "Through-the-wall tomographic imaging using chirp signals," in Proceedings of the IEEE International Symposium on Antennas and Propagation, pp. 2091-2094, Spokane, Wash, USA, July 2011.

[28] K. A. Lukin, P. L. Vyplavin, V. V. Kudriashov, V. P. Palamarchuk, P. G. Sushenko, and N. K. Zaets, "Radar tomography using noise waveform, antenna with beam synthesis and MIMO principle," in Proceedings of the 9th International Conference on Antenna Theory and Techniques (ICATT '13), pp. 190-192, Odessa, Ukraine, September 2013.

[29] G. Fornaro, A. Pauciullo, D. Reale, and S. Verde, "Multilook SAR tomography for sensing built structures with very high resolution spaceborne sensors," in Proceedings of the 11th European Radar Conference (EuRad '14), pp. 221-224, Rome, Italy, October 2014.

[30] A. Broquetas, R. Jordi, J. M. Rius, A. R. Elias-Fuste, A. Cardama, and L. Jofre, "Cylindrical geometry: a further step in active microwave tomography," IEEE Transactions on Microwave Theory and Techniques, vol. 39, no. 5, pp. 836-844, 1991.

[31] H. C. Rhim and O. Büyüköztürk, "Wideband microwave imaging of concrete for nondestructive testing," Journal of Structural Engineering, vol. 126, no. 12, pp. 1451-1457, 2000.

[32] P. Mojabi and J. LoVetri, "A prescaled multiplicative regularized Gauss-Newton inversion," IEEE Transactions on Antennas and Propagation, vol. 59, no. 8, pp. 2954-2963, 2011.

[33] A. J. Devaney, "Inversion formula for inverse scattering within the Born approximation," Optics Letters, vol. 7, no. 3, pp. 111-112, 1982.

[34] T. H. Chu and K. Y. Lee, "Wide-band microwave diffraction tomography under Born approximation," IEEE Transactions on Antennas and Propagation, vol. 37, no. 4, pp. 515-519, 1989.

[35] B. S. Robinson and J. F. Greenleaf, "An experimental study of diffraction tomography under the Born approximation," in Acoustical Imaging, H. Lee and G. Wade, Eds., vol. 18 of Acoustical Imaging, pp. 391-400, Springer, New York, NY, USA, 1990. 
[36] L. Turner, "The evolution of featureless waveforms for LPI communications," in Proceedings of the IEEE National Aerospace and Electronics Conference (NAECON '91), pp. 1325-1331, Dayton, Ohio, USA, May 1991.

[37] C.-P. Lai and R. M. Narayanan, "Ultrawideband random noise radar design for through-wall surveillance," IEEE Transactions on Aerospace and Electronic Systems, vol. 46, no. 4, pp. 17161730, 2010.

[38] H. J. Shin, R. M. Narayanan, and M. Rangaswamy, "Simulations of tomographic imaging of various target scenarios using noise waveforms," in Proceedings of the IEEE International Radar Conference, pp. 963-968, IEEE, Arlington, Va, USA, May 2015.

[39] L. Jofre, A. P. Toda, J. M. J. Montana et al., "UWB shortrange bifocusing tomographic imaging," IEEE Transactions on Instrumentation and Measurement, vol. 57, no. 11, pp. 2414-2420, 2008.

[40] D. Sheen, D. McMakin, and T. Hall, "Near-field threedimensional radar imaging techniques and applications," Applied Optics, vol. 49, no. 19, pp. E83-E93, 2010.

[41] S. Gu, C. Li, X. Gao, Z. Sun, and G. Fang, "Terahertz aperture synthesized imaging with fan-beam scanning for personnel screening," IEEE Transactions on Microwave Theory and Techniques, vol. 60, no. 12, pp. 3877-3885, 2012.

[42] R. F. Harrington, "On scattering by large conducting bodies," IRE Transactions on Antennas and Propagation, vol. 7, no. 2, pp. 150-153, 1959.

[43] H. J. Shin, R. M. Narayanan, and M. Rangaswamy, "Ultrawideband noise radar imaging of impenetrable cylindrical objects using diffraction tomography," International Journal of Microwave Science and Technology, vol. 2014, Article ID 601659, 22 pages, 2014.

[44] W. Tabbara, B. Duchêne, C. Pichot, D. Lesselier, L. Chommeloux, and N. Joachimowicz, "Diffraction tomography: contribution to the analysis of some applications in microwaves and ultrasonics," Inverse Problems, vol. 4, no. 2, pp. 305-331, 1988.

[45] J. C. Bolomey and C. Pichot, "Microwave tomography: from theory to practical imaging systems," International Journal of Imaging Systems and Technology, vol. 2, no. 2, pp. 144-156, 1990.

[46] H. J. Shin, R. M. Narayanan, and M. Rangaswamy, "Diffraction tomography for ultra-wideband noise radar and imaging quality measure of a cylindrical perfectly conducting object," in Proceedings of the IEEE Radar Conference (RadarCon '14), pp. 702-707, Cincinnati, Ohio, USA, May 2014.

[47] H. J. Shin, R. M. Narayanan, and M. Rangaswamy, "Ultrawideband noise radar imaging of cylindrical PEC objects using diffraction tomography," in Radar Sensor Technology XVIII, vol. 9077 of Proceedings of SPIE, pp. 1-10, Baltimore, Md, USA, May 2014.

[48] A. M. Eskicioglu and P. S. Fisher, "Image quality measures and their performance," IEEE Transactions on Communications, vol. 43, no. 12, pp. 2959-2965, 1995.

[49] I. Avcibaş, B. Sankur, and K. Sayood, "Statistical evaluation of image quality measures," Journal of Electronic Imaging, vol. 11, no. 2, pp. 206-223, 2002.

[50] H. J. Shin, M. A. Asmuth, R. M. Narayanan, and M. Rangaswamy, "Principle and experimental results of ultrawideband noise radar imaging of a cylindrical conducting object using diffraction tomography," in Radar Sensor Technology XIX; and Active and Passive Signatures VI, vol. 9461 of Proceedings of SPIE, pp. 1-11, Baltimore, Md, USA, April 2015.
[51] M. G. Amin, Through-the-Wall Radar Imaging, CRC Press, Boca Raton, Fla, USA, 2010.

[52] M. A. Asmuth, H. J. Shin, R. M. Narayanan, and M. Rangaswamy, "Design and implementation of a noise radar tomographic system," in Radar Sensor Technology XIX, vol. 9461 of Proceedings of SPIE, pp. 1-9, Baltimore, Md, USA, April 2015. 


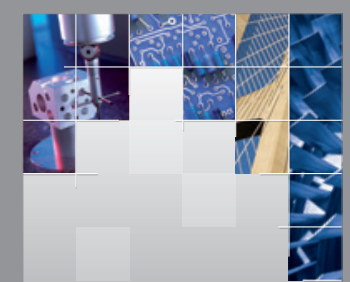

\section{Enfincering}
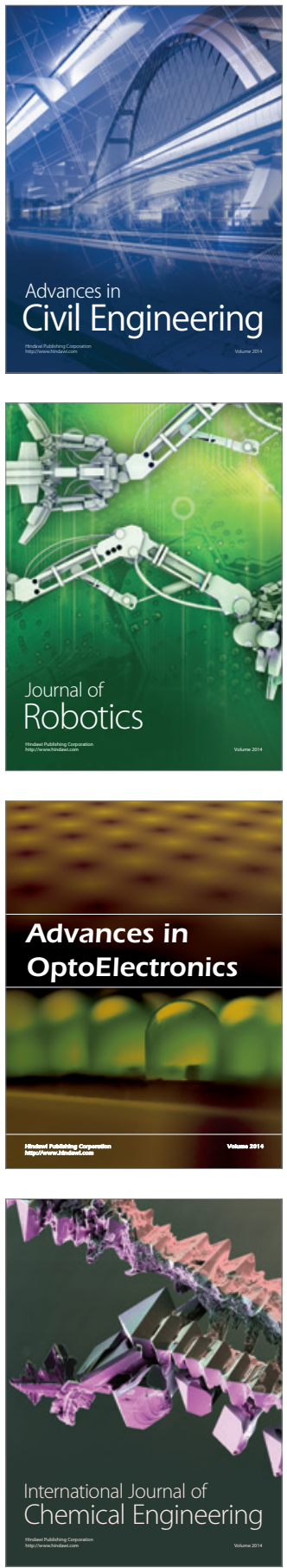

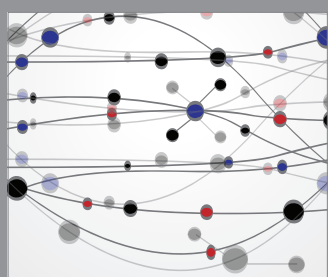

The Scientific World Journal

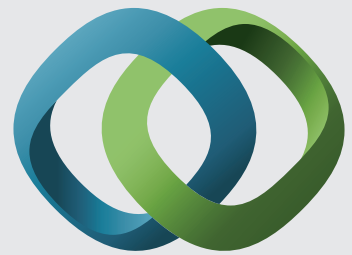

\section{Hindawi}

Submit your manuscripts at

http://www.hindawi.com
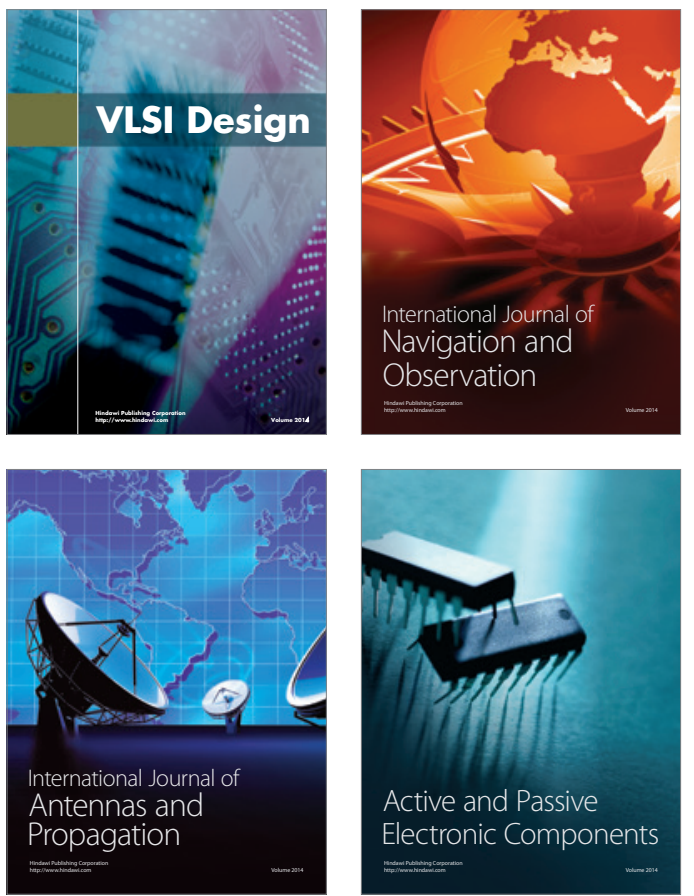
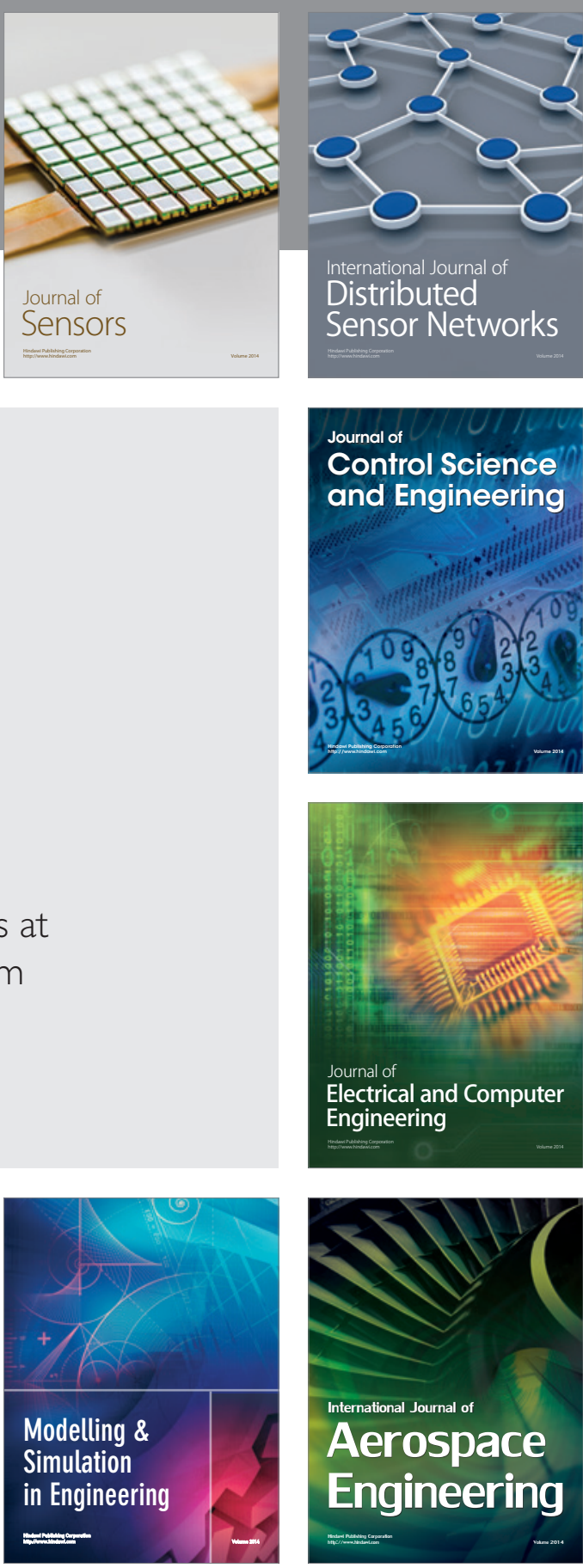

International Journal of

Distributed

Sensor Networks

Journal of

Control Science

and Engineering
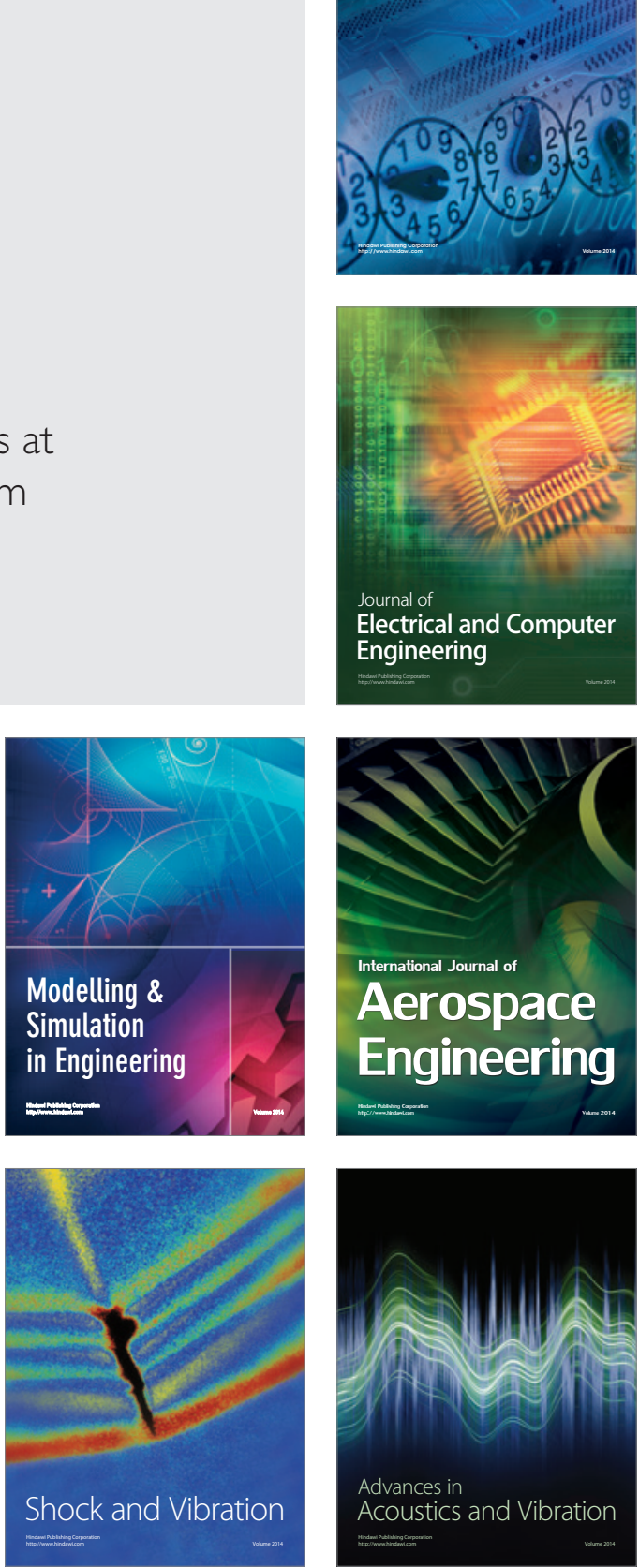